\title{
On the intrinsic timescales of temporal variability in measurements of the surface solar radiation
}

\author{
Marc Bengulescu, Philippe Blanc, and Lucien Wald \\ MINES ParisTech, PSL Research University, Centre for Observation, Impacts, \\ Energy CS 10207 - 06904 Sophia Antipolis CEDEX, France
}

Correspondence: P. Blanc (philippe.blanc@mines-paristech.fr)

Received: 13 July 2016 - Discussion started: 17 October 2016

Revised: 28 September 2017 - Accepted: 10 December 2017 - Published: 26 January 2018

\begin{abstract}
This study is concerned with the intrinsic temporal scales of the variability in the surface solar irradiance (SSI). The data consist of decennial time series of daily means of the SSI obtained from high-quality measurements of the broadband solar radiation impinging on a horizontal plane at ground level, issued from different Baseline Surface Radiation Network (BSRN) ground stations around the world. First, embedded oscillations sorted in terms of increasing timescales of the data are extracted by empirical mode decomposition (EMD). Next, Hilbert spectral analysis is applied to obtain an amplitude-modulation-frequencymodulation (AM-FM) representation of the data. The timevarying nature of the characteristic timescales of variability, along with the variations in the signal intensity, are thus revealed. A novel, adaptive null hypothesis based on the general statistical characteristics of noise is employed in order to discriminate between the different features of the data, those that have a deterministic origin and those being realizations of various stochastic processes. The data have a significant spectral peak corresponding to the yearly variability cycle and feature quasi-stochastic high-frequency variability components, irrespective of the geographical location or of the local climate. Moreover, the amplitude of this latter feature is shown to be modulated by variations in the yearly cycle, which is indicative of nonlinear multiplicative cross-scale couplings. The study has possible implications on the modeling and the forecast of the surface solar radiation, by clearly discriminating the deterministic from the quasistochastic character of the data, at different local timescales.
\end{abstract}

\section{Introduction}

The power of the electromagnetic radiation from the Sun that reaches the surface of the Earth is estimated at around $10^{17} \mathrm{~W}$. Thus, solar irradiance is the main driver behind the weather and climate systems on the planet. As such, the Global Climate Observing System (GCOS) program has identified the surface solar irradiance (SSI) as an Essential Climate Variable that helps understand climate evolution and guides adaptation and mitigation efforts (Bojinski et al., 2014). Long-term time series of the SSI are instrumental in engineering and finance by enabling, for example, the optimal determination of geographical sites for solar power plants and guiding investment decisions, respectively (Schroedter-Homscheidt et al., 2006). Thus, better knowledge of the SSI and of its temporal variability, as recorded in long-term time series, is one of the intents of this work.

Temporally, the SSI exhibits a very wide dynamic range. Its short-term timescales of variability, such as clouds briefly obscuring the Sun, are observed over seconds. At the opposite scale, thousands or even millions of years are to be used, as related to the change of the orbital parameters of the Earth-Sun system or to stellar evolution (Beer et al., 2006). In spite of this large span of characteristic scales of temporal variability, most of the studies dealing with this physical quantity have focused primarily on a few selected timescales of interest. As such, reports have either dealt with global averages and long-term trends (Trenberth et al., 2009; Wahab et al., 2010; Pachauri et al., 2014; Blanc et al., 2015), have only scrutinized the short-term, high-frequency variability (Yordanov et al., 2013; Lauret et al., 2016), or have focused exclusively on a few intermediate scales (Coskun et al., 2011; Medvigy and Beaulieu, 2012). Although considerably differ- 
M. Bengulescu et al.: Intrinsic timescales of surface solar radiation

ing in methods, taken together the previously cited studies add valuable contributions to our knowledge of the SSI. But is it possible to analyze the variability in the SSI across multiple timescales in a unitary way?

To do so, first a decomposition of the time series into uncorrelated sub-constituents with distinct characteristic timescales should be preferred. Analysis would then ensue in a like manner for each scale. The timescales, or characteristic periods of a time series, can be identified with the inverse of the frequency at which the processes that generate the data occur. It then follows that methods portraying the changes of the spectral content of a time series with respect to time are potentially good candidates. This would enable both the identification of the periodicities and of the dynamic evolution of the processes generating the data. A general class of useful signal processing techniques can thus be identified in the so-called time-frequency distributions that depict the intensity (or energy) of a signal in the time and the frequency domains simultaneously (Cohen, 1989). Such methods are commonly employed for geophysical signal processing (Tary et al., 2014).

Another factor to be taken into account is the nonlinear and non-stationary characteristics of the measured solar radiation data (Zeng et al., 2013). Handling such data issued from the nonlinear interaction of physical processes, often also found under the influence of non-stationary external forcings, calls for an adaptive data analysis approach (Wu et al., 2011).

The study at hand will make use of the Hilbert-Huang transform (HHT), an adaptive, data-driven analysis technique designed specifically for investigating nonlinear and nonstationary data (Huang et al., 1998). The HHT adaptively decomposes any dataset into basis functions that are derived solely from the local properties of the time series. A time-frequency-energy representation of the data is then constructed from these basis functions. The HHT has seen extensive use in geophysical signal analysis and spectral estimation (Solé et al., 2007; Huang and Wu, 2008; Vecchio et al., 2010; Lee and Ouarda, 2011; Alberti et al., 2014; Huang and Shen, 2014; Tary et al., 2014). The HHT has also been previously employed on SSI datasets (Duffy, 2004; Calif et al., 2013; Bengulescu et al., 2016a, b). A similar technique has been independently proposed by Nagovitsyn (1997) for the analysis of the nonlinear, non-stationary, long-range solar activity. In this light, the use of the HHT for the study of the temporal variability in the SSI appears to be appropriate. The inner workings of this data processing method are detailed in a dedicated subsection.

Regardless of the methods used, when analyzing data there is always the need to discriminate between deterministic signals and what are assumed to be background stochastic realizations (Rios et al., 2015). The classical way to solve this when employing the HHT on geophysical signals, such as the SSI, is to presume some model for the background power spectrum, against which the identified features are then compared (Huang and Wu, 2008; Franzke, 2009, 2012).
Table 1. Ground measurement stations listing.

\begin{tabular}{lllrrl}
\hline Code & Location $^{\mathrm{a}}$ & & Latitude $^{\mathrm{b}}$ & Longitude $^{\mathrm{b}}$ & Climate $^{\mathrm{c}}$ \\
\hline BOU & Boulder & (US) & 40.0500 & -105.0070 & BSk \\
CAR & Carpentras & (FR) & 44.0830 & 5.0590 & Csa \\
PAY & Payerne & (CH) & 46.8150 & 6.9440 & Cfb \\
TAT & Tateno & (JP) & 36.0581 & 140.1258 & Cfa \\
\hline
\end{tabular}

a Country codes according to ISO $3166-1$ alpha-2.

b Positive north for latitude and positive east for longitude, following ISO 19115.

${ }^{c}$ Köppen-Geiger climate classification according to Kottek et al. (2006).

In contrast, the present study parts with the traditional approach, by adopting a novel, adaptive null hypothesis introduced by Chen et al. (2013) that requires no a priori knowledge of the nature of the background processes; further discussion thereof will be presented in due course. A somewhat similar objective can be found in the work of Rios and de Mello (2016), though their method of discrimination between stochastic and deterministic components is fundamentally different. Kolotkov et al. (2016) also propose a method for discriminating frequency-dependent stochastic components by empirically estimating their power law spectral energy distribution and respective confidence bounds. Approaches for discriminating high-frequency fluctuations from large timescale modulations are also described by Flandrin et al. (2004b) and Alberti et al. (2016).

At this point, the general outline of our study can be summarized as follows. We analyze measurements of daily means of SSI at different geographical locations. We focus on identifying and analyzing the intrinsic modes of the temporal variability in the SSI, as revealed by the HHT. We also investigate the physical and statistical significance of these modes. We show that the HHT is able to discriminate between a deterministic yearly cycle and multiple high-frequency (quasi-)stochastic components. We also find a non-null, statistically significant rank correlation between the amplitude envelopes of the high-frequency scales and the yearly cycle. We then discuss the possible implications of our findings on the modeling and forecast of the SSI.

The study is organized as follows. Section 2 discusses the data sources and the preprocessing. In Sect. 3 the adaptive data analysis approach is described. Section 4 will present the results obtained, with the discussion thereof being deferred to Sect. 5. Conclusions and outlook are presented in Sect. 6. Code and data availabilities are indicated in the Code and Data availability sections, respectively. Lastly, acknowledgements and a bibliographical list conclude the study.

\section{Data sources and preprocessing}

The data under scrutiny in this study consist of 10-year time series of daily means of SSI obtained from high-quality measurements performed at four different locations (Table 1 and Fig. 1). The measurement stations are part of the Baseline 


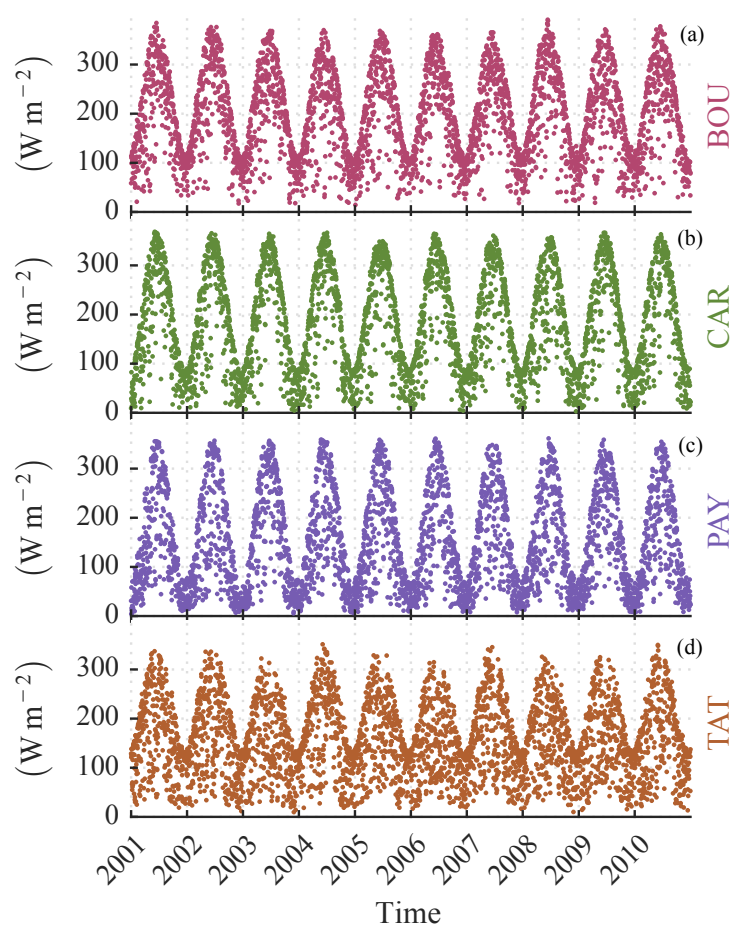

Figure 1. The four decennial SSI time series investigated in this study, spanning 2001 through 2010. From top to bottom: BOU, CAR, PAY, and TAT. Each point corresponds to a daily mean of SSI. Time markers on the abscissa indicate the start of the corresponding year.

Surface Radiation Network (BSRN), a worldwide radiometric network providing accurate readings of the SSI at $1 \mathrm{~min}$ temporal resolution and with an uncertainty requirement at $5 \mathrm{~W} \mathrm{~m}^{-2}$ (Ohmura et al., 1998).

The four time series for the period 2001-2010 have been quality checked according to Roesch et al. (2011). Next, daily means of SSI were then calculated from these raw time series only if more than $80 \%$ of the data during daylight were valid. Lastly, any isolated missing daily means were completed by linear interpolation applied to the daily clearness index, $K_{\mathrm{T}}$, which is the ratio between the daily mean of SSI and the daily mean of the total solar irradiance (TSI) received on a horizontal surface at the top of atmosphere for the same geographical coordinates.

Two measuring stations are located in Europe, one in Japan, and one in North America in order to capture various climatic conditions. Boulder (hereafter abbreviated as BOU) experiences a midlatitude steppe, cool type of climate (Köppen-Geiger: BSk), while at Carpentras (abbreviated as CAR) the climate is a humid subtropical, Mediterranean one (Köppen-Geiger: Csa). Both sites experience many sunny days during the year. As a rule of thumb, $K_{\mathrm{T}}$ equal to $0.2-0.3$ denotes cloudy, overcast conditions, while $K_{\mathrm{T}}$ around 0.7 indicates sunny conditions. Figure 2 exhibits the histograms of $K_{\mathrm{T}}$ for the four stations. One may observe the high frequen-

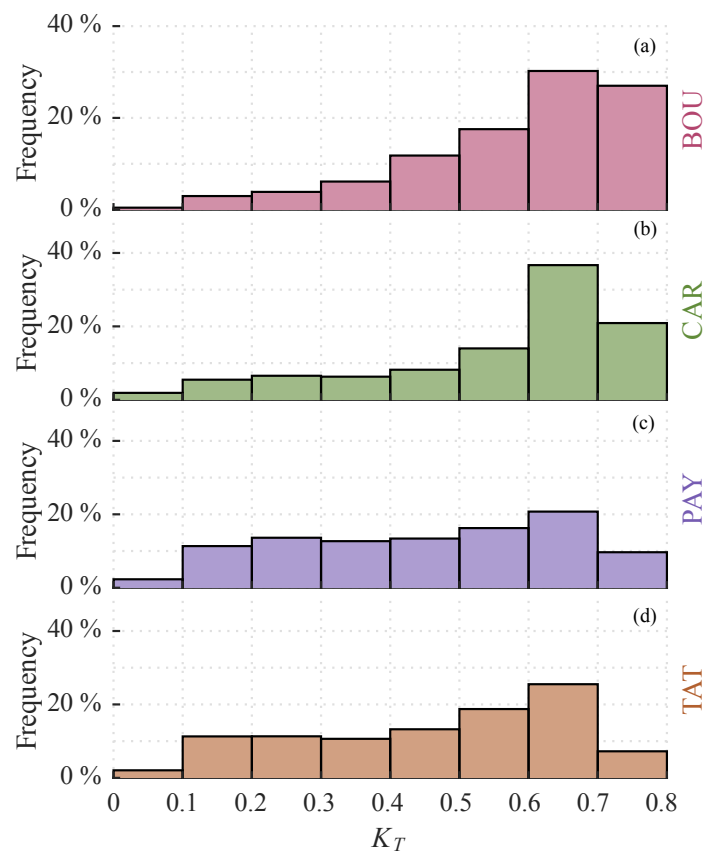

Figure 2. Histograms of the daily clearness index $K_{\mathrm{T}}$ over the decennial time span in percent frequency. From top to bottom: BOU, CAR, PAY, and TAT.

cies of the greatest values of $K_{\mathrm{T}}$ for BOU and CAR. The median $\widetilde{K}_{\mathrm{T}}$ is equal to 0.63 for both BOU and CAR, which means that half of the days exhibit $K_{\mathrm{T}}$ greater than 0.63 . The climate in Payerne (PAY) is classified as a marine west coast, mild climate (Köppen-Geiger: $\mathrm{Cfb}$ ), and Tateno (TAT) has a humid subtropical, east coast climate (Köppen-Geiger: Cfa). Compared to BOU and CAR, PAY and TAT exhibit more uniform histograms, with less days with cloud-free conditions, and experience more overcast and broken clouds conditions. The median $\widetilde{K}_{\mathrm{T}}$ is equal to 0.47 for PAY and 0.51 for TAT. Except for TAT, which is embedded in an urban setting, the stations are located in rural environments; the local topography for BOU and TAT is flat with grassy surfaces, while for CAR and PAY the area is hilly with cultivated surfaces (BSRN, 2015).

Any further reference to seasons and seasonal phenomena shall be understood as occurring in the Northern Hemisphere since the stations are situated at boreal latitudes.

\section{Adaptive data analysis}

Ideally, data analysis methods should require that no assumptions be made about the nature of the scrutinized time series, i.e., neither linearity nor stationarity should be presumed. This is because the true character of the underlying processes that have generated the data is usually not known beforehand. Adaptivity to the analyzed data would also be a sought-after feature, in the sense of not imposing a set of 
patterns against which data would be decomposed, but rather letting the data themselves drive the decomposition. This latter criterion ensures both that the extracted components carry physical meaning and that the influence of the mathematical artifacts inherent to the method on the rendered picture of temporal variability is kept to a minimum (Wu et al., 2011). Since such a decomposition is only determined by the local characteristic timescales of the data, its appropriateness to nonlinear and non-stationary time-series analysis is immediate (Huang et al., 1998).

\subsection{The Hilbert-Huang transform}

The Hilbert-Huang transform (HHT) is an adaptive data analysis technique built with the previous consideration in mind. It involves two distinct steps - the empirical mode decomposition (EMD) followed by Hilbert spectral analysis. In-depth discussion of each step is carried out within the dedicated subsections that follow.

\subsubsection{The empirical mode decomposition}

The first step of the HHT is the empirical mode decomposition (EMD), an algorithmic procedure in essence, by which oscillations that present a common local timescale are iteratively extracted from the data. These oscillatory components of the data are called intrinsic mode functions (IMFs). An IMF is any function that satisfies two criteria: (1) its number of extrema and zero crossings differs at most by one and (2) at any data point the mean value of its upper and lower envelopes is zero. These two properties ensure that IMFs have a well-behaved Hilbert transform (Huang et al., 1998). Owing to the adaptive nature of the EMD, the IMFs represent the basis functions onto which the data are projected during decomposition. This is in contrast with the Fourier or wavelet transforms where the basis functions are fixed in advance (Huang and $\mathrm{Wu}, 2008)$. Once all the IMFs have been extracted, all that is left of the time series is a residue, or trend, which cannot be mathematically thought of as an oscillation at the span of the data. A sketch of the EMD algorithm is provided in Algorithm 1.

Lines 6-12 of the EMD algorithm represent the so-called "sifting loop" which has a two-fold purpose - to discard any riding waves and to render the IMFs more symmetric. The stoppage criterion for the sifting loop is closely related to how the latter controls the filter character of the EMD. On the one hand, an infinite number of sifting iterations would asymptotically approach the result of the Fourier decomposition (i.e., constant amplitude envelopes) (Wang et al., 2010). On the other hand, several studies performed on time series of pure noise (Flandrin and Gonçalvès, 2004; Flandrin et al., 2004a; Wu and Huang, 2004) have shown the decomposition behaves like an adaptive "wavelet-like" dyadic filter if the number of sifting iterations is kept small, around 10, which also assures maximum component separation and minimum leakage (Wu and Huang, 2010). This stoppage criterion of 10 sifting iterations is currently the recommended one for practical applications (Wu and Huang, 2009, 2010) and is also the one employed in the study.

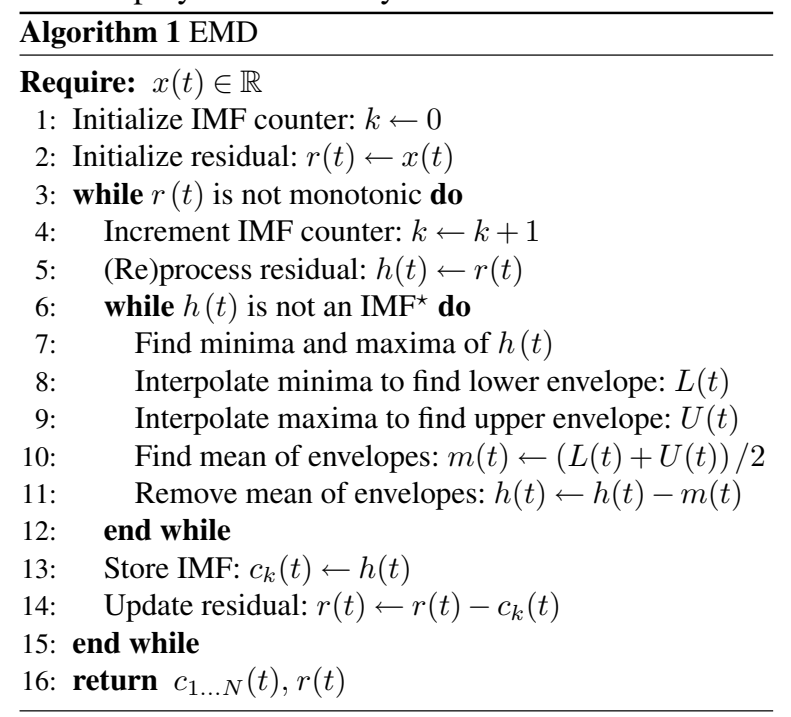

* See text for the definition of IMF. This "sifting" loop should be run approximately 10 times (Wu and Huang, 2009, 2010).

It also worth noting that the preferred interpolation method in the EMD, i.e., lines 8 and 9 of Algorithm 1, are cubic splines (Rilling et al., 2003). Because of oscillations of these interpolating splines edge effects may appear in the EMD but are usually contained within a half-period of a component at data boundaries (Wu et al., 2011).

One of the drawbacks of the original EMD is that it may introduce a phenomenon known as "mode mixing". This is the manifestation of oscillations with dissimilar timescales in the same IMF or the presence of oscillations with similar timescales in different IMFs. A workaround was proposed by $\mathrm{Wu}$ and Huang (2009) with ensemble empirical mode decomposition (EEMD). The idea was to run the decomposition over an ensemble of copies of the original signal to which white Gaussian noise has been added, with the final result obtained by averaging. Although the EEMD improved the mode-mixing problem, the different sums of signal and noise produced different numbers of modes, making the final averaging somewhat difficult. Added to this, the reconstructed signal still contained some residual noise and thus was not identical to the original. To overcome this situation, Torres et al. (2011) have proposed another iteration of the EMD, the complete EEMD with adaptive noise (CEEMDAN). This method also decomposes the white noise into modes, along with the signal, such that at each stage of the decomposition a particular noise is added and a unique residue is computed to obtain each mode. However, the modes of CEEMDAN still contain some residual noise and sometimes spurious modes appear in the early stages of the decomposition. The next iteration of the method, the improved complete ensemble EMD (ICEEMD or ICEEMDAN), overcomes these issues by fixing the signal-to-noise 


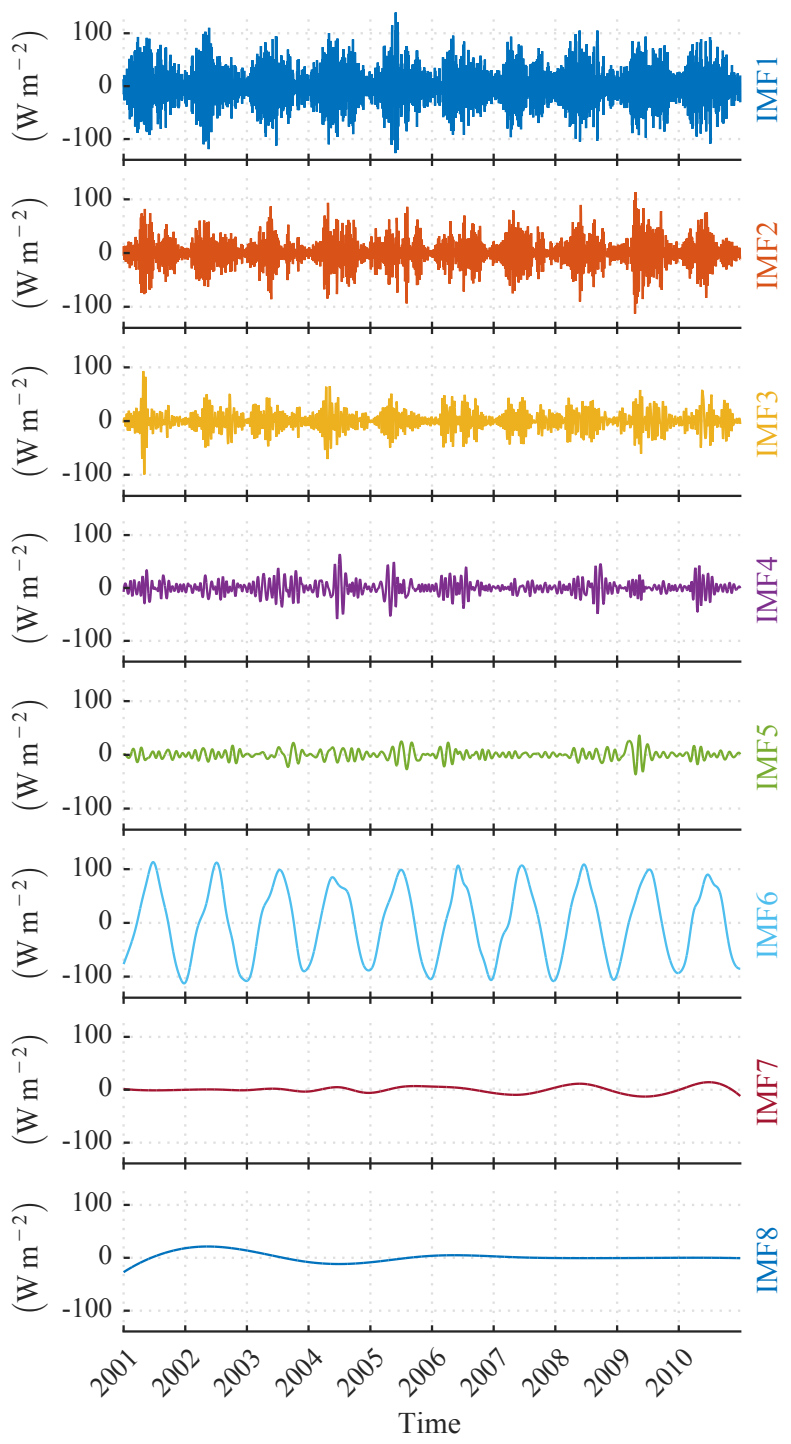

Figure 3. The eight IMFs obtained by decomposing the BOU time series; from top to bottom: IMF1-IMF8. The panels plot SSI (ordinate) versus time (abscissa). Time markers on the horizontal axes indicate 1 January of the corresponding year. The zero-centered oscillatory nature of the modes can be clearly seen. Also apparent is the local timescale increase with mode number.

ratio for all stages of the decomposition process (Colominas et al., 2014). The ICEEMD method is that used in this study. In addition, a fast EMD routine provided by Wang et al. (2014) has been used to decrease the computation time.

To illustrate the workings of the EMD, the eight IMFs of the BOU time series are presented in Fig. 3 in the order they were obtained, from top to bottom. As EMD operates in the time domain, the IMFs have the same temporal support as the original data and, by construction, upper and lower amplitude envelopes that are symmetrical with respect to zero. It can be observed in Fig. 3 that, as the decomposition progresses, the timescale of the IMFs increases; i.e., the intrin-

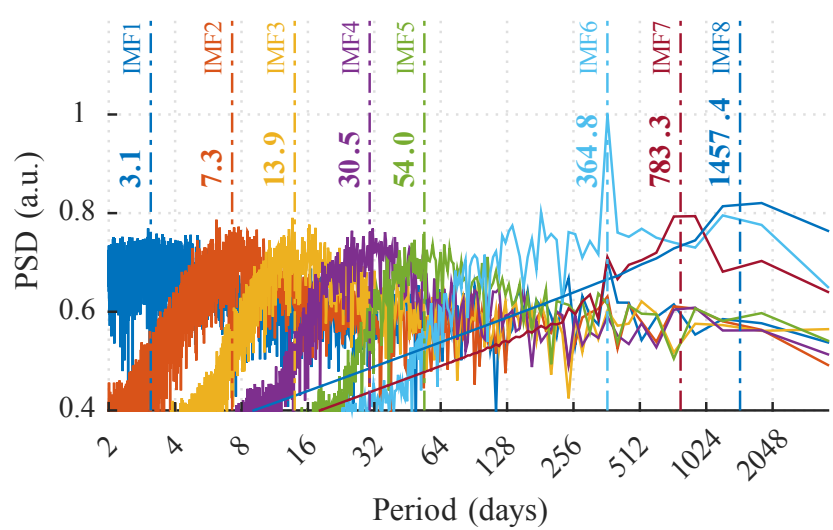

Figure 4. The power spectral density (PSD) of the eight IMFs for BOU (solid line) on a logarithmic scale normalized with respect to the power of the highest spectral peak. The period, or inverse frequency, runs on the abscissa in a base- 2 logarithm. The individual spectra are shown in the same colors as the IMFs from Fig. 3; from left to right: IMF1-IMF8. The Fourier estimates of the median periods, marked along the dash-dotted lines, are seen to increase with mode number. Notable features are the prominent spectral peak of IMF6 at $\sim 365$ days corresponding to the yearly cycle and the apparent dyadic repartition of the timescales for IMF1-IMF5.

sic oscillations are getting further spaced apart with increasing IMF number. Another view of this is brought by Fig. 4, where the power spectral density (PSD) and a Fourier estimate of the mean period of each IMF are plotted. To aid the reader, the colors used to portray the individual IMF spectra are the same as for the time-domain representation from Fig. 3. The spectral shapes of the IMF1-IMF5 are similar in form, i.e., bell curves, and their median periods roughly follow a dyadic scale, i.e., doubling with increasing IMF number as 3.1 days $\rightarrow 7.3$ days $\rightarrow 13.9$ days $\rightarrow 30.5$ days $\rightarrow$ 54.0 days. This doubling of the timescale for these first five IMFs is the hallmark output of an efficient dyadic filter. Subsequently, it is shown that this dyadic repartition is pertinent to identifying deterministic signals from random realizations of quasi-stochastic background processes. This finding is even more interesting, since the median periods have been estimated with a Fourier-based method, which measures the period globally over the whole time range of the IMFs. In contrast, a measure of the local period in the Hilbert sense is a much better estimate, since it has an accuracy as low as a quarter wavelength of temporal resolution with respect to the average timescale of the IMF (Huang et al., 2009).

\subsubsection{Hilbert spectral analysis}

Once the empirical mode decomposition is completed, the second and last step of the HHT consists in the Hilbert spectral analysis of the previously obtained IMFs. Each IMF and its Hilbert transform are used to construct a complex analytic signal, described by an amplitude-modulation-frequency- 
modulation (AM-FM) model. This decomposition into two time-varying parts corresponding, respectively, to instantaneous amplitude and instantaneous frequency is very useful for the purpose of this study. It enables the identification, in a time-varying sense, of how much power (i.e., the square of amplitude) occurs at which timescale (i.e., the inverse of frequency).

The Hilbert transform of each real-valued IMF $c_{k}(t)$ can be written as

$\sigma_{k}(t)=\mathcal{H}\left(c_{k}(t)\right)=\frac{1}{\pi} P \int_{-\infty}^{\infty} \frac{c_{k}(\tau)}{t-\tau} \mathrm{d} \tau$,

where subscript $k$ designates the $k$ th IMF and $P$ indicates the Cauchy principal value. From each IMF and its Hilberttransformed version, a unique complex-valued analytic signal can be obtained (Gabor, 1946):

$z_{k}(t)=c_{k}(t)+i \cdot \sigma_{k}(t)=a_{k}(t) \cdot e^{i \cdot \theta_{k}(t)}$,

in which

$a_{k}(t)=\sqrt{c_{k}^{2}(t)+\sigma_{k}^{2}(t)}$

is the instantaneous amplitude and

$\theta_{k}(t)=\tan ^{-1}\left(\frac{\sigma_{k}(t)}{c_{k}(t)}\right)$

is the instantaneous phase. The instantaneous frequency is the first time derivative of the instantaneous phase:

$\omega_{k}(t)=\frac{1}{2 \pi} \frac{\mathrm{d} \theta_{k}(t)}{\mathrm{d} t}$.

Figure 5 provides a visual guide to this concept by illustrating the AM-FM decomposition of IMF5 for the BOU time series. The top panel (IMF5) of the figure reproduces the mode function, which is also the real part of the analytic signal from Eq. (2). The amplitude of the latter (AM), given in Eq. (3), which is the envelope of the original signal, is then extracted and plotted in the second panel. This amplitude is not a constant, but rather a time-dependent function. Next, by removing the AM component from the signal through simple division, the frequency modulation component is obtained, i.e., the complex exponential in Eq. (2); the real part of this component (FM) is plotted in the third panel. The FM is a trigonometric function with a phase argument that is a time-dependent function, as seen from Eq. (4). The local frequency is then just the first temporal derivative of this phase, as defined in Eq. (5). The inverse of the local frequency, i.e., the local timescale of the signal, is depicted in the bottom panel (timescale), where its temporal variability can be clearly distinguished. Owing to their time-varying character, the amplitude and frequency are usually encountered in the literature under the terms instantaneous amplitude and instantaneous frequency, respectively.

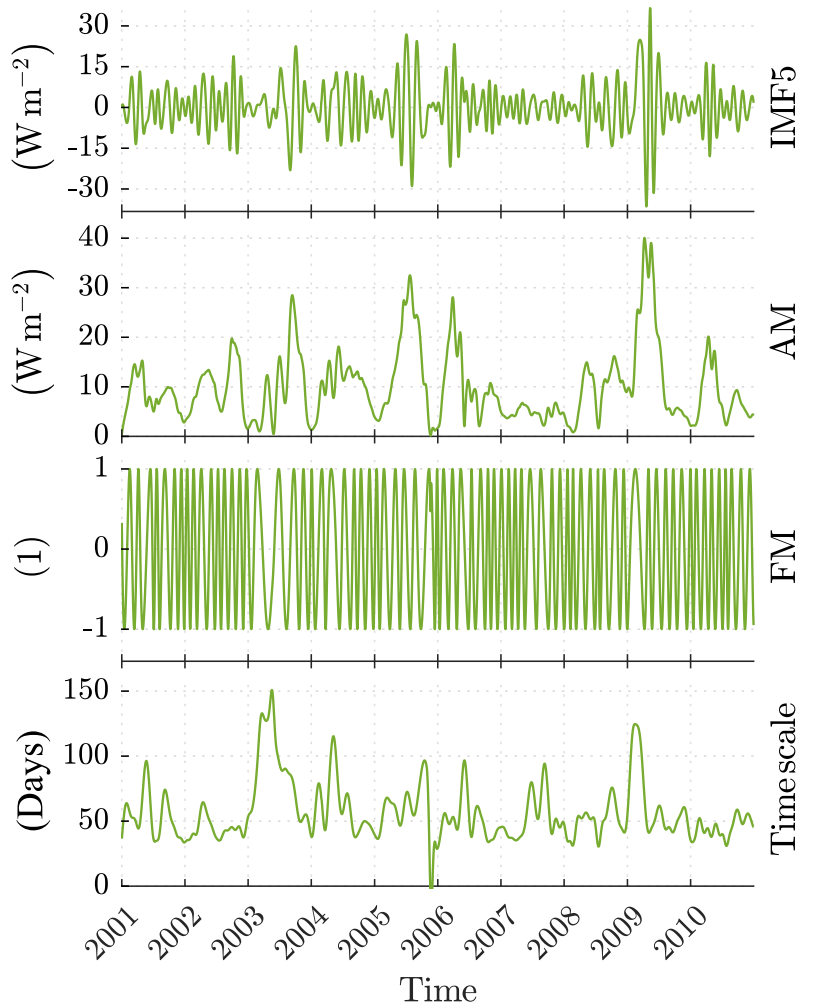

Figure 5. Hilbert spectral analysis of the fifth IMF of the BOU time series. The intrinsic mode function (IMF5 panel) is the product of its constituent slowly varying amplitude-modulation part (AM panel) and of its rapidly changing frequency-modulation component (FM panel). The time-varying local timescale, extracted from the FM component, is also depicted (timescale panel). Time markers on the abscissa denote the beginning of the corresponding year.

The original time-series $x(t)$ can then be expressed as a sum of AM-FM signals riding onto the EMD trend, $r(t)$, as follows:

$x(t)=\operatorname{Re}\left[\sum_{k=1}^{N} a_{k}(t) \cdot e^{i \int \omega_{k}(\tau) \mathrm{d} \tau}\right]+r(t)$.

The square of the instantaneous amplitude and the instantaneous frequency of the IMFs can then be used to represent the data as an energy density distribution overlaid on the time-frequency space, as in Eq. (7). This representation, called the Hilbert energy spectrum, is defined by Huang et al. (2011) as "the energy density distribution in a time-frequency space divided into equal-sized bins of $\Delta t \times \Delta \omega$ with the value in each bin summed and designated as $a^{2}(t)$ at the proper time, $t$, and proper instantaneous frequency, $\omega$ ".

$S(\omega, t)=\sum_{k=1}^{N} a_{k}^{2}(t) \cdot e^{i \sum \omega_{k}(t)}$ 


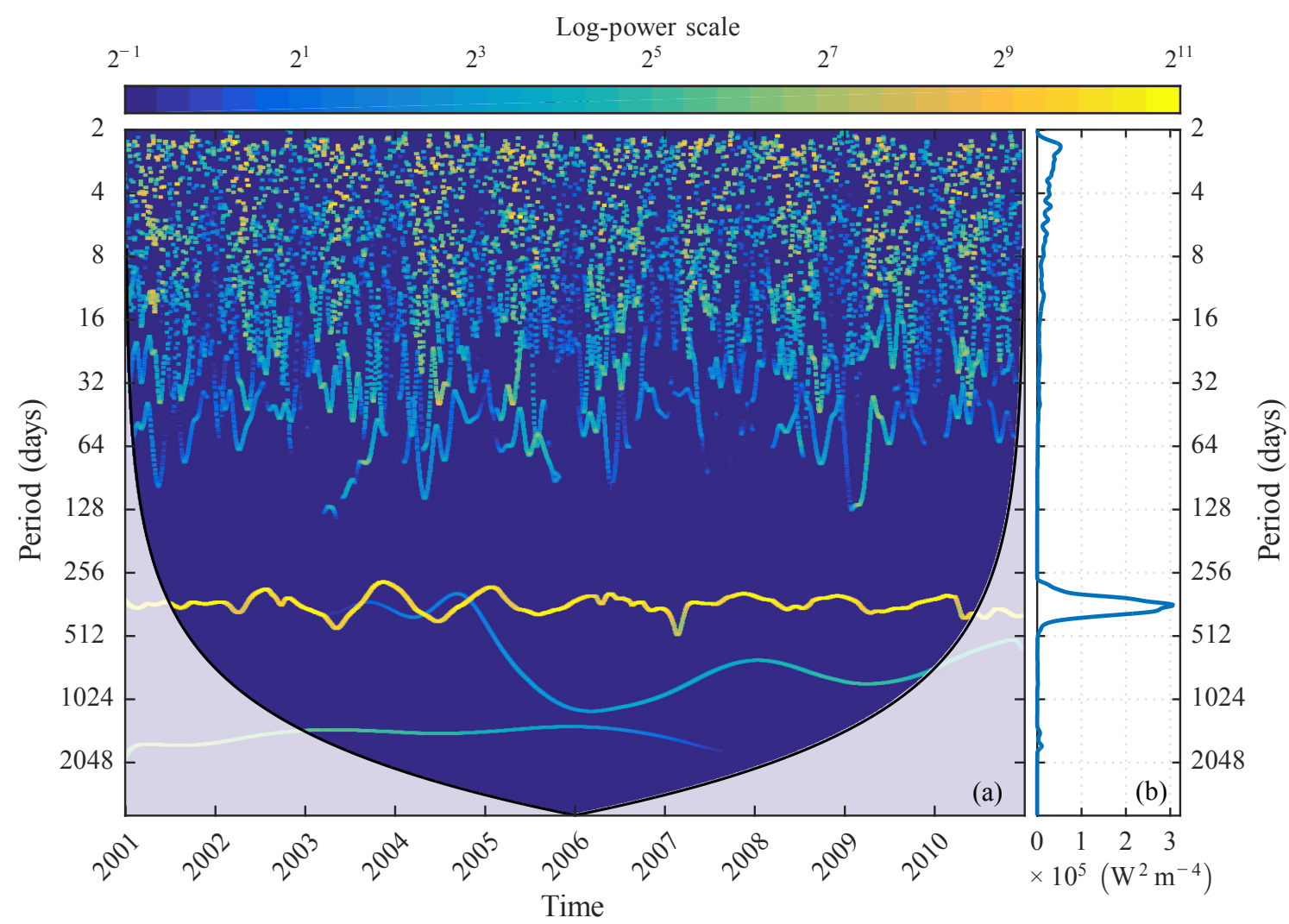

Figure 6. The Hilbert spectrum (a) of the 10-year time series of SSI for BOU, spanning 2001 through 2010. Pixel color encodes power (logarithmic-scale color bar on top) at each instant (abscissa) and each scale (ordinate). Time markers on the horizontal axis denote the start of the corresponding year. The whited-out area indicates the regions where edge effects become significant. The Hilbert marginal spectrum in the panel (b) is the time-integrated version, i.e., line-by-line sum, of the Hilbert spectrum and indicates the amount of power at each scale.

The time-integrated version of Eq. (7), the Hilbert marginal spectrum $S_{\mathrm{M}}(\omega)$, is similar, but not identical, to the traditional Fourier spectrum:

$$
S_{\mathrm{M}}(\omega)=\int_{0}^{T} S(\omega, t) \mathrm{d} t .
$$

An example of Hilbert spectral representation is given in Fig. 6a where the BOU time series is shown as an energy density distribution over-imposed on a time-frequency space as in Eq. (7). Each pixel in the Hilbert spectrum is identified by three attributes - color, abscissa, and ordinate - through which it denotes the local power (color, log scale) of the corresponding time series, at a certain time (abscissa) and at a certain timescale (ordinate, log scale). For the sake of readability, the spectrum is binned in time, scale, and color space and has been smoothed. Hence, some aliasing may occur. Some features may be represented as continuous lines while others are rendered as point-like, especially where rapid frequency modulation takes place, such as in the high-frequency bands.

Interpretation of Hilbert spectral features at data boundaries must be done with care due to possible oscillations of the spline interpolants used in the EMD (see Algorithm 1). This effect is similar to the "cone of influence" in the popular wavelet transform (Torrence and Compo, 1998). With the EMD, edge effects are usually contained within a half-period of a component at data boundaries (Wu et al., 2011). In Fig. 6 this region has been whited out.

The plot in Fig. $6 \mathrm{~b}$ is the Hilbert marginal spectrum, or the time-integrated variant of the image at its left, indicating the amount of power at each timescale. This time-agnostic representation is comparable, but not identical, to the Fourier spectrum of the same time series. It should be once again emphasized that the Hilbert marginal spectrum is obtained from local features of the data, with its components having instantaneous amplitude and instantaneous frequency, as opposed to the global outlook of the Fourier spectrum whose constituents have constant amplitude and constant frequency throughout the whole domain.

\subsection{Adaptive background null hypothesis}

Which confidence can be attributed to the information extracted by the EMD? More specifically, how can one ascertain that a certain IMF is the result of a real physical process 
as opposed to it possibly being a stochastic manifestation of background processes?

In the past, several investigations have been carried out in order to identify the effects of the EMD when applied to time series issued from various models, such as white, red, or fractional Gaussian noise, (Huang et al., 2003; Flandrin and Gonçalvès, 2004; Flandrin et al., 2004a, 2005; Wu and Huang, 2004; Rilling et al., 2005; Schlotthauer et al., 2009; Colominas et al., 2012). As a result, it has been consistently shown that, irrespective of the assumed noise model, the EMD acts as an efficient wavelet-like dyadic filter, decomposing the stochastic inputs into IMFs having the same spectral shape but that are shifted in the frequency domain.

Nevertheless, the rejection of a null hypothesis based on an a priori assumed model of the background does not preclude the probability that the now statistically significant deemed signals originate from a stochastic process of a different kind. Furthermore, as the EMD is an adaptive, data-driven decomposition, it would be desirable to also employ a null hypothesis that shares the same characteristics, making no beforehand assumptions about the character of the background processes.

Following P. Flandrin (personal communication, 2015) and Chen et al. (2013) this study will make use of the robust statistical properties of the EMD with respect to a wide class of background models in order to adaptively contrast potential signals against presumed stochastic realizations, as detailed hereafter. Owing to its dyadic filter character, the EMD decomposes noise time series into IMFs having similar spectral shape but that are translated to roughly the next lower octave in the spectral domain. When the sampling step is increased, i.e., the sampling frequency is reduced by fractionally resampling the input, these components cannot preserve their original locations in the spectral domain and are instead shifted towards lower frequencies. Hence, significance testing of IMFs is done by verifying if the IMF remains unchanged in the time-frequency representation of the signal during fractional resampling of the latter.

A Hilbert marginal spectrum $S_{\mathrm{M}_{k}}(\omega)$ is first constructed for each IMF from its instantaneous amplitude $a_{k}(t)$ and instantaneous frequency $\omega_{k}(t)$. Next the spectrum-weighted mean frequency (SWMF) $\bar{\omega}_{k}$ of each IMF is computed (Chen et al., 2013):

$\bar{\omega}_{k}=\frac{\int S_{\mathrm{M}_{k}}(\omega) \omega \mathrm{d} \omega}{\int S_{\mathrm{M}_{k}}(\omega) \mathrm{d} \omega}$.

Then, the time series is fractionally resampled by making the original sampling rate $\Delta t$ progressively larger, i.e., the time spacing of the data points becomes

$\Delta t_{l}=\Delta t \cdot l, \quad l \in\{1.1,1.2, \ldots, 1.9\}$.

For each sampling rate $l$ and for each IMF $k$, the SWMFs are then recomputed, obtaining a set $\bar{\omega}_{k, l}$. To enhance the visibility of the evolution of frequency as a function of the resampling rate, normalization is performed as in

$\widehat{\omega}_{k, l}=\frac{\bar{\omega}_{k, l}}{\bar{\omega}_{k, 1}}$,

with $\bar{\omega}_{k, 1}$ being the SWMFs of the modes of the data having the original sampling rate. Therefore, the normalized SWMFs for the IMFs of the original data will be unity, i.e., $\widehat{\omega}_{k, 1}=1, \forall k$.

Since the EMD is an efficient dyadic filter, frequency deviation from the unity line will occur for IMFs generated by stochastic processes. It follows that when $\widehat{\omega}_{k, l} \simeq 1, \forall l$, the null hypothesis that mode $k$ is the realization of stochastic processes can be rejected.

\section{Results}

The IMFs obtained from the BOU time series from Fig. 3 have already served as an illustrative example on the operation of the EMD. The IMFs for the other datasets (not shown) are very similar and are discussed in the following. It must be noted that, like BOU, the CAR time series is decomposed into 8 IMFs, while the PAY data have 9, and 10 IMFs are obtained for TAT. Besides the IMFs, for each time series the decomposition also yields a residual, or trend (also not shown). With respect to the decennial time span of the analysis (10 years), the trend can be thought of as a lowpass approximation of the data (Moghtaderi et al., 2013), but not as an oscillation. Since this work focuses mostly on the characteristic scales of temporal variability, the EMD trends along with their statistical significance and physical meaning do not fall within the scope of the study; for such discussion, see for example Franzke (2012).

From the Fourier spectra of the IMFs in Fig. 4 it can be seen that, owing to its median period of 364.8 days, IMF6 can be unambiguously associated with the yearly cycle, as dictated by the orbital parameters of the Earth-Sun system. IMF6 also accounts for the most prominent visual feature in the original data (Fig. 1a: BOU), with its maxima and minima denoting summer and winter, respectively. Further evidence is brought by the spectral shape of IMF6, distinguished by a sharp peak that has the largest power in Fig. 4. Also noteworthy is that IMF6 seems to modulate the previous five IMFs, as these latter seem to exhibit amplitude excursions that are approximately in phase with the amplitude of IMF6, a phenomenon that is most visually distinguishable in Fig. 3 for the first three IMFs during the year 2005.

Finally, the last two components, IMF7 and IMF8, having median periods of 783.3 and 1457.4 days (Fig. 4), respectively, are seen to exhibit only slight amplitude deviation from zero in their temporal representation. Moreover, these fluctuations in amplitude occur at the end of the signal for IMF7 and at the front edge for IMF8. Interpretation of these components should, thus, be done with care, since edge effects for the EMD are known to be usually contained within 


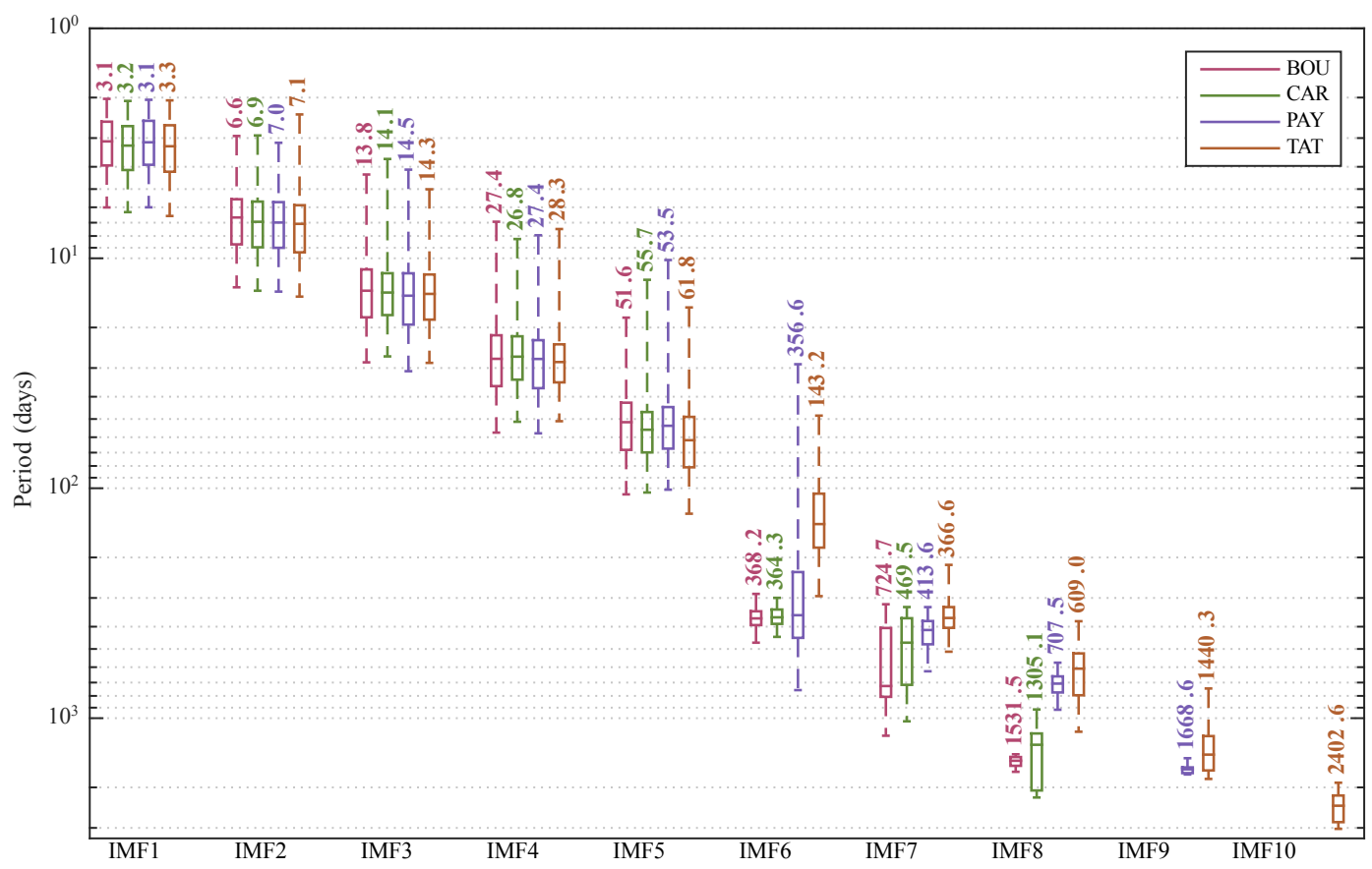

Figure 7. Box plot of the instantaneous timescales of the IMFs for the four stations. The top and the bottom edges of the boxes represent the first (Q1) and, respectively, the third (Q3) quartiles. The bars inside boxes denote the second quartile (Q2), i.e., the median. The whisker length is set at at most 1.5 times the interquartile range, i.e., $1.5 \times(\mathrm{Q} 3-\mathrm{Q} 1)$; hence, the whiskers roughly correspond to \pm 2.7 standard deviations, or equivalently $\backsim 99 \%$ of the data, assuming normal distribution. The median for each box is expressed numerically above the lower whiskers. Outliers are omitted. Numeric values for all the statistical descriptors are shown in Table 2.

a half-period of a component at data boundaries (Wu et al., 2011), i.e., approximately 1 year for IMF7 and 2 years for IMF8.

With the FM components obtained, it becomes possible to illustrate the frequency contents of each time series in terms of its individual IMFs, as shown in Fig. 7, where by means of box plots the distribution of the local timescale of each mode is conveyed. This box plot representation is somehow incomplete, as it only accounts for the period distribution of the modes and does not take into account either the amplitude or the temporal localization of the events. For readability, the characteristic period of each IMF with its range of variability is also shown numerically in Table 2. The box plots of the instantaneous amplitude of each IMF are given in Fig. 8.

For all time series, IMF1-IMF5 have very similar median periods (Fig. 7) that approximate the dyadic sequence: 3.5 days $\rightarrow 7$ days $\rightarrow 14$ days $\rightarrow 28$ days $\rightarrow 56$ days. This dyadic repartition of their median timescales is worthy of attention since, as it is apparent in Sects. 3.2 and 5.1, it plays a major role in discriminating which IMFs can be attributed to deterministic phenomena as opposed to being the output of random realizations of background processes. Moreover, besides the notable similarity among the medians of these modes, for all the datasets both the interquartile ranges and the total ranges of these first five modes exhibit approximately the same variability. Added to this, IMF6 for BOU,
CAR, and PAY, as well as IMF7 for TAT, whose median periods are, respectively, 368.2, 364.3, and 356.6, as well as 366.6 days, can clearly be associated with the yearly cycle given by the revolution of the Earth around the Sun. This yearly component is very similar for BOU, CAR, and to a lesser extent TAT, with an interquartile range that is concentrated around almost the same median value and with the only minor difference being the slightly extended range for TAT of 300 days as opposed to 200 days for the other two. The PAY yearly mode differs from those of the other stations, with its interquartile range and foremost its range being much larger, the latter even overlapping the interquartile ranges of IMF5 and IMF4. This is a result of the mode-mixing phenomenon described in Sect. 3.1 that may arise with the EMD, i.e., the coexistence or mixing of different timescales in the same IMF, mainly related to the intermittence of signal and to contamination with noise (Huang et al., 2003). Nevertheless, the spectral part of IMF6 which overlaps IMF5 and IMF4 has very low power (Bengulescu et al., 2016b); thus, this phenomenon does not influence the validity of the analysis. With this in mind, one notes that for BOU and CAR no spectral components are present in the 100- to 300-day band. Furthermore, TAT is the only dataset that has a transitional mode of 143.2 days, with the median period in between the first five IMFs common to all stations and the yearly cycle. 
Table 2. Statistical descriptors of the instantaneous timescales of the IMFs, expressed in days.

\begin{tabular}{|c|c|c|c|c|c|c|c|c|c|c|c|}
\hline Station & Descriptor* & IMF1 & IMF2 & IMF3 & IMF4 & IMF5 & IMF6 & IMF7 & IMF8 & IMF9 & IMF10 \\
\hline \multirow[t]{5}{*}{$\mathrm{BOU}$} & Lower whisker & 2.0 & 2.9 & 4.3 & 6.9 & 18.1 & 288.1 & 319.6 & 1435.5 & & \\
\hline & First quartile & 2.5 & 5.5 & 11.2 & 21.6 & 42.5 & 342.9 & 405.4 & 1476.0 & & \\
\hline & Second quartile & 3.1 & 6.6 & 13.8 & 27.4 & 51.6 & 368.2 & 724.7 & 1531.5 & & \\
\hline & Third quartile & 3.9 & 8.7 & 18.1 & 36.0 & 68.1 & 393.7 & 807.6 & 1611.1 & & \\
\hline & Upper whisker & 6.0 & 13.4 & 28.3 & 57.3 & 106.3 & 469.5 & 1192.1 & 1710.9 & & \\
\hline \multirow[t]{5}{*}{ CAR } & Lower whisker & 2.1 & 2.9 & 3.7 & 8.2 & 12.4 & 299.7 & 328.6 & 916.4 & & \\
\hline & First quartile & 2.7 & 5.7 & 11.6 & 21.8 & 46.6 & 337.0 & 367.6 & 1165.4 & & \\
\hline & Second quartile & 3.2 & 6.9 & 14.1 & 26.8 & 55.7 & 364.3 & 469.5 & 1305.1 & & \\
\hline & Third quartile & 4.1 & 9.0 & 17.7 & 33.7 & 69.8 & 388.9 & 716.2 & 2062.3 & & \\
\hline & Upper whisker & 6.3 & 13.8 & 26.7 & 51.4 & 104.4 & 443.0 & 1031.8 & 2212.8 & & \\
\hline \multirow[t]{5}{*}{ PAY } & Lower whisker & 2.0 & 3.1 & 4.1 & 7.9 & 10.2 & 28.9 & 328.8 & 573.7 & 1493.0 & \\
\hline & First quartile & 2.5 & 5.7 & 11.6 & 22.7 & 44.4 & 231.5 & 378.1 & 658.0 & 1637.0 & \\
\hline & Second quartile & 3.1 & 7.0 & 14.5 & 27.4 & 53.5 & 356.6 & 413.6 & 707.5 & 1668.6 & \\
\hline & Third quartile & 3.9 & 9.0 & 19.4 & 36.7 & 67.3 & 447.4 & 477.2 & 772.9 & 1733.5 & \\
\hline & Upper whisker & 6.0 & 14.0 & 31.0 & 57.7 & 101.5 & 755.0 & 625.3 & 918.9 & 1757.2 & \\
\hline \multirow[t]{5}{*}{ TAT } & Lower whisker & 2.1 & 2.4 & 5.0 & 7.5 & 16.4 & 48.3 & 215.4 & 378.8 & 742.8 & 1908.4 \\
\hline & First quartile & 2.6 & 5.9 & 11.8 & 23.7 & 48.9 & 105.6 & 328.7 & 522.8 & 1196.3 & 2169.2 \\
\hline & Second quartile & 3.3 & 7.1 & 14.3 & 28.3 & 61.8 & 143.2 & 366.6 & 609.0 & 1440.3 & 2402.6 \\
\hline & Third quartile & 4.2 & 9.4 & 18.5 & 34.6 & 81.1 & 181.3 & 404.7 & 795.0 & 1687.8 & 2831.9 \\
\hline & Upper whisker & 6.5 & 14.7 & 28.5 & 51.1 & 129.0 & 294.6 & 513.9 & 1145.5 & 1837.4 & 3029.0 \\
\hline
\end{tabular}

* A box plot illustration of the statistical descriptors is shown in Fig. 7.

At this point, the Hilbert frequency distribution of the IMFs for BOU may be compared to the Fourier one from the PSD in Fig. 4. As previously mentioned, the Hilbert estimates are based on local features of the data and thus are more accurate than the Fourier ones when applied to nonstationary signals. This can be seen especially when comparing the range of the first five high-frequency IMFs, which is upper bounded to about 100 days in Fig. 7, whereas in the PSD from Fig. 4 the spectra of the same components are seen to span the whole timescale range. This also holds for IMF6, which has very narrow Hilbert period range, whose Fourier analogue is the sharp peak in the PSD of the same mode. Similar statements can be made for IMF7 and IMF8. To sum up, it is found that, while the Hilbert period distributions of the modes have compact supports, the Fourier representations of the same components span the whole frequency range. Nevertheless, most of the power in the Fourier PSD is assigned to a frequency band that closely corresponds to the Hilbert range. Owing to the global nature of the Fourier transform, however, additional spectral coefficients are needed to provide a complete mathematical description of the data.

Resuming the discussion of the IMF timescales from Fig. 7, it can be observed that the low-frequency, i.e., greater than 1 year, variability in the data, trend notwithstanding, is assigned into slightly overlapping (within the same time series) IMFs that span the spectrum starting from the 1-year mark. For BOU and CAR time series, there are only two modes extending beyond 1 year. First, IMF7 can be seen to span approximately the same range for both these stations, from about 1 year to slightly more than 3 years. For BOU, however, the interquartile range and especially the median period is shifted towards higher periods, i.e., 724.7 days vs. 469.5 days for CAR. The last modes of IMF8 of these stations are very different, with a very narrow range around the median of 1531.5 days for BOU, as well as a range of 900 to over 2000 days and a median of 1305.1 days for CAR. For the PAY data, the low-frequency components have narrower spectral support, with two IMFs (IMF7 and 8) that cover the band from 1 to 2.5 years and median periods of 413.6 and 707.5 days, as well as the IMF9 around 4.5 years ( $\sim 1668$ days) with a very narrow range. It must also be noted that IMF7 for BOU, CAR, and PAY has the same lower-end support, and that the couple (IMF7, IMF8) of PAY taken together somehow emulates IMF7 for BOU and CAR. Lastly, TAT is the only dataset whose the low-frequency variability is expressed by three components, IMF8-IMF10, with mean periods of $609,1440.3$, and 2402.6 days. While the first quartile of IMF9 coincides with the upper range of IMF8, the upper range of IMF9 is slightly below the lower range of IMF10; hence, the last two modes do not overlap at all. By its range, IMF8 of TAT approximates IMF7 for BOU and CAR, but there is no proximity in terms of median or interquartile range. Similarly, the IMF9 of TAT resembles the IMF8 of CAR in terms of range, but their medians are not in close agreement and their interquartile ranges even less so. 


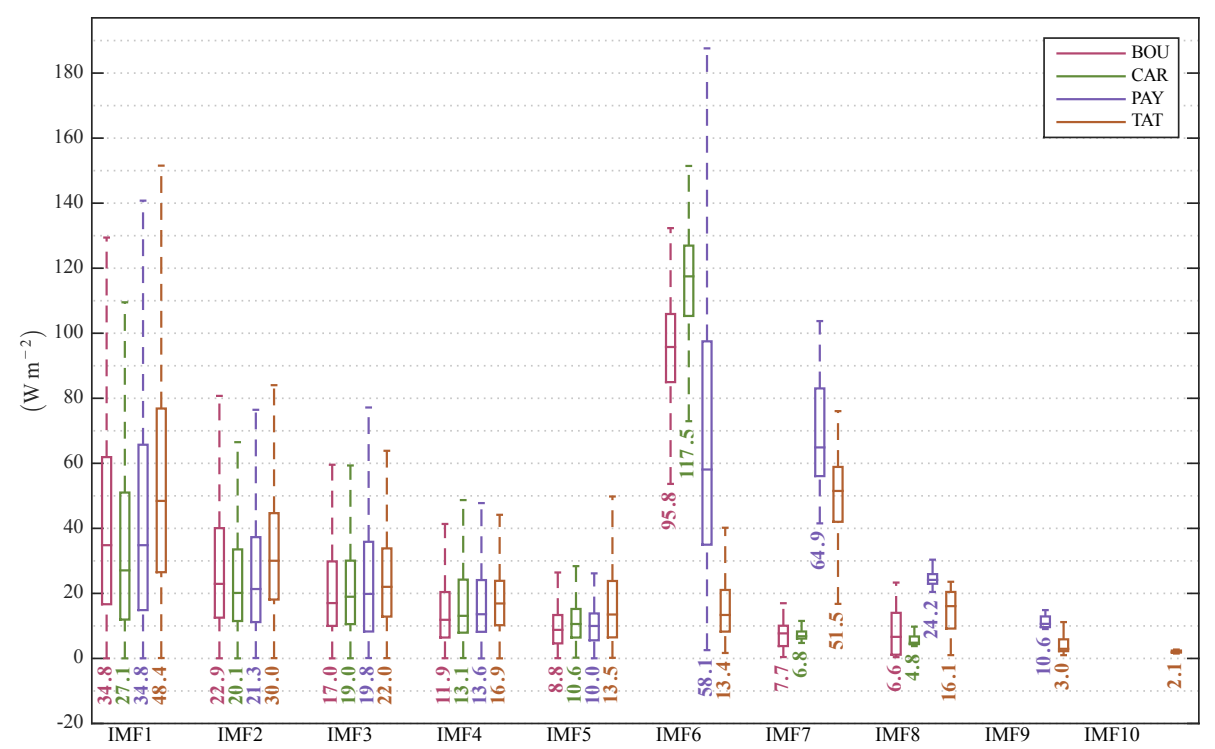

Figure 8. Box plot of the instantaneous amplitudes of the IMFs for the four stations. The bottom and the top edges of the boxes represent the first (Q1) and, respectively, the third (Q3) quartiles. The bars inside boxes denote the second quartile (Q2), i.e., the median. The whisker length is set at at most 1.5 times the interquartile range, i.e., $1.5 \times(\mathrm{Q} 3-\mathrm{Q} 1)$; hence, the whiskers roughly correspond to \pm 2.7 standard deviations, or equivalently $\backsim 99 \%$ of the data, assuming normal distribution. The median for each box is expressed numerically below the lower whiskers. Outliers are omitted.

With the scrutiny of these low-frequency components, the discussion of the timescale distribution of the IMFs from Fig. 7 can now be concluded. However, as previously mentioned, this particular illustration, although instructive, is incomplete. First, the box plot representation does not take the instantaneous variations in frequency into account, but renders global aggregates instead - much like the traditional Fourier methods, with the interquartile range spread in addition. This is done on purpose, with the intent of making it easier for the readership not accustomed to the HHT to create analogies with the more familiar methods (e.g., Fourier analysis, wavelets). Second and last, this particular representation is totally devoid of any information pertaining to the local amplitude, or power, or variance, of the data. With these considerations in mind, the Hilbert spectrum, a true timefrequency representation for nonlinear and non-stationary data, is discussed next. Since the goal of this exercise is to lay the groundwork for the forthcoming discussion, only the spectrum for the BOU data is provided as an example.

The BOU Hilbert spectrum from Fig. 6 exhibits a highfrequency feature between 2 and $\backsim 100$ days, which corresponds to first five IMFs of the time series. The instantaneous timescales of these modes overlap (Fig. 7), hence the appearance on the Hilbert spectrum of a continuum instead of distinct bands. This spectral feature has relatively low power, which decreases with increasing period, as can be inferred from the sloped dent in the marginal Hilbert spectrum corresponding to this region. In the 2- to 32-day band, amplitude modulation by the yearly cycle can be inferred from the periodic change in color, with yellow-green tones, oc- curring mostly during the high irradiance regime of summer, that turn blue during the winterly minima. Next, in the band between 100 and 300 days, a gap in the spectrum is apparent, as can also be inferred from the lack of support in this region for any of the BOU IMFs in Fig. 7. The yellow trace, corresponding to IMF6, exhibits frequency modulation around the 1-year period, seen as oscillations in the range of 300 to 450 days, which is also the support of this mode in Table 2. The color of this IMF indicates that it has the highest power of all the components, as can also be inferred from the large peak on the marginal spectrum. The corresponding timescale fluctuations are centered in 365 days, and frequency modulation is greatest during 2003 through 2005. From 2006 onwards, however, frequency modulation is less pronounced - perhaps capturing the low solar activity around the 2008 minimum in the 11-year cycle solar cycle (Hathaway, 2015). The final two low-frequency, blue-green traces on the spectrum correspond to IMF7 and IMF8. For IMF7, mode mixing is apparent through the occasional sharing of the yearly timescale band with IMF6, between mid2003 and 2005. IMF7 has such low power that it fails to leave an imprint on the marginal spectrum and it seems to suddenly spring into existence during summer 2003, which is in perfect agreement with its temporal representation from Fig. 3 (panel IMF7), whence it can be seen to have negligible amplitude during the first 2.5 years. Also in agreement with its temporal depiction from Fig. 3 (panel IMF8), IMF8 starts out in light-green hues and slowly vanishes during mid2007. Although this last BOU mode manages to register on the marginal spectrum through two very slight indentations 
around 1500 days (which is about the median period of this mode from Figs. 4 and 7), most of its power lies within edge effect territory, hence interpretation of these slight bumps is ambiguous at best.

Thus far, all time series have been shown to share a highfrequency constituent between 2 and 100 days composed of five IMFs with mean periods following a dyadic sequence, and an IMF around 365 days that captures the yearly variability. For BOU, CAR, and PAY, a low-power region can be found in the 100- to 300-day band. Beyond the 1 year timescale, the low-frequency variability in the 1.5 - to 6-year band is captured by another two (BOU and CAR) or three (PAY and TAT) components. The TAT data are the only time series that has an IMF in the low-power band between the high-frequency feature and the yearly cycle (median period 143.2 days).

\section{Discussion}

The previously identified features of the SSI time series will now be discussed in terms of their intrinsic temporal scales of variability and physical statistical meaning.

\subsection{Discriminating deterministic signals from stochastic components in the IMFs}

At this point, having identified the spectral characteristics of the SSI time series by means of the HHT, a question arises with regard to their physical and statistical significance, namely how can one ascertain which features represent the expression of real, deterministic physical phenomena and which ones can be attributed to random realizations of background processes. Such a method, proposed by Chen et al. (2013), is described in Sect. 3.2. The procedure was applied to the first eight IMFs of all the time series and the results are presented in Fig. 9 (from top to bottom: BOU, CAR, PAY, and TAT). First, each time series was resampled with a fractional sampling rate up to a factor of 2; i.e., the original uniform time spacing of the data, $\Delta t$, was progressively made larger and larger, as described in Eq. (10): $\Delta t_{l}=\Delta t \cdot l$, where $l \in\{1.1,1.2, \ldots, 1.9\}$ is the resampling rate and runs along the horizontal axis. Next, the HHT was used to decompose the resulting time series into IMFs and to compute their spectrum-weighted mean frequencies, following Eq. (9). In order to emphasize the effects of the fractional resampling on the spectral contents of the IMFs, these latter frequencies were then normalized by the SWMF of the original, non-resampled data as per Eq. (11). For each dataset, this ratio is indicated on the $y$ axis, as $\widehat{\omega}_{k, l}$, with $k \in\{1 \ldots 8\}$ indicating the IMF number. It then becomes possible to follow the evolution of the normalized SWMF of each individual IMF as a function of the fractional resampling rate (Fig. 9). As the EMD is an efficient wavelet-like dyadic filter, it follows that the IMFs of time series of random pro- cesses undergo a translation towards lower frequencies under fractional resampling. Therefore, for those IMFs whose SWMFs are not downshifted during resampling, the null hypothesis that they are purely stochastic can be rejected, i.e., they represent meaningful signals. Stated otherwise, an IMF $k$ is deemed not to be stochastic in nature if its normalized SWMFs $\widehat{\omega}_{k, l}$ stay close to the unity line for all $l$. From Fig. 9 it can be observed that for all the stations the only component that maintains a quasi-constant frequency under fractional resampling is the mode representative of the yearly variability, i.e., IMF6 for BOU, CAR, and PAY, as well as IMF7 for TAT. All the other IMFs experience the previously described frequency downshifting; hence, for them the null hypothesis that they are purely stochastic in nature cannot be rejected. Since the normalized SWMFs of the yearly components clearly stray from the black dashed line in Fig. 9, the result that they are not stochastic in nature is unambiguous. This also indicates that the signal-to-noise ratio of these components is well above the minimum value of $0.2-0.3$ required to reveal potential signals (Chen et al., 2013).

At this point, several precautionary notes are compulsory. First, the rule of inference used here is modus tollens; i.e., the results from Fig. 9 do not imply that the modes which experience downshift in their SWMFs are made up of pure noise. It is subsequently shown that, for the first five IMFs at least, this is indeed the case; although (quasi-)stochastic in nature, they are not completely devoid of information. Second, the result is mostly qualitative, since it is difficult to define a confidence interval owing to the adaptive nature of the null hypothesis that can account for different types of models of the stochastic background. Third and last, the approach is best applied only to the high-frequency modes, with respect to the data length and sampling, since by resampling spurious low-frequency oscillation may inadvertently be introduced (Chen et al., 2013). This is further supported by the fact that, as the IMF number progresses, the region where the influence of edge effects becomes important gets larger and larger, hence only adding uncertainty to the interpretation of the results. This is also the reason why this type of analysis was only carried out on the first eight IMFs of each dataset.

\subsection{Amplitude modulation through nonlinear cross-scale coupling}

This section investigates whether the first five IMFs can be modeled as purely uncorrelated, random noise or whether they also contain any other form of information. To test this, the rank correlation between the yearly and sub-yearly IMFs and their envelopes, e.g., the AM part in the middle panel of Fig. 5, has been computed for each SSI time series. Kendall's rank correlation coefficient, $\tau$, a statistical measure of ordinal association describing how similar the orderings of the data are when ranked (Kendall, 1938), is employed here to establish whether each pair of the two variables, AM $x$ and IMF $y$ with $x, y \in\{1 \ldots 7\}$, may be regarded or not as independent. 

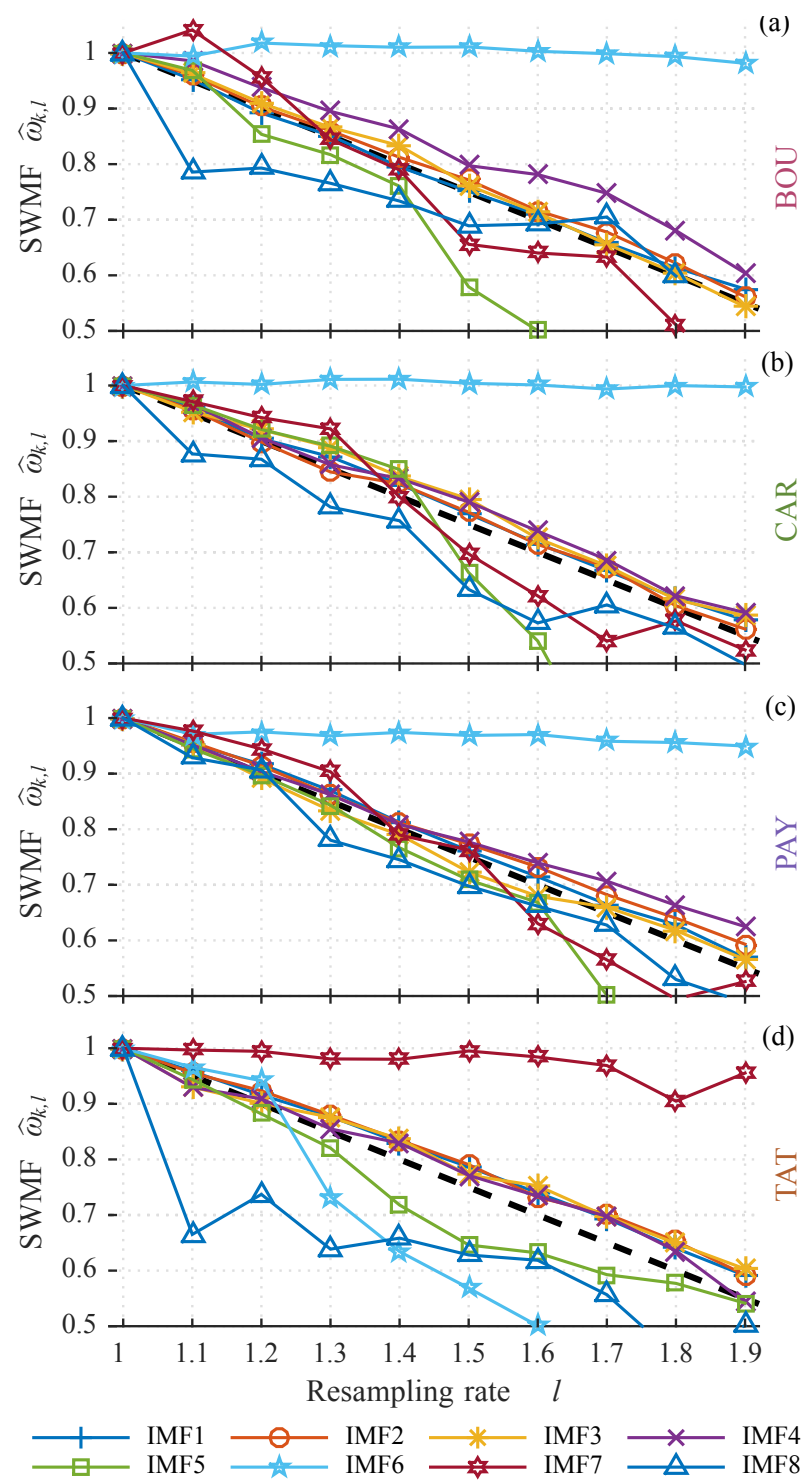

Figure 9. The drift of normalized SWMF $\widehat{\omega}_{k, l}$ (ordinate) of IMF $k$, with $k \in\{1 \ldots 8\}$, as a function of the resampling rate $l$ (abscissa) for the four time series. From top to bottom: BOU, CAR, PAY, and TAT. The black dashed diagonal depicts the behavior of a pure noise time series under an ideal dyadic filter. For all datasets, the only mode that maintains a quasi-constant frequency under fractional resampling is the IMF associated with the yearly cycle, i.e., IMF6 for BOU, CAR, and PAY and IMF7 for TAT. In all the other IMFs quasi-stochastic behavior is apparent through frequency downshifting towards the next lower octave, approximately following the dashed line.

$\tau=1$ indicates perfect agreement between rankings, while $\tau=-1$ denotes perfect disagreement - i.e., one ranking is the reverse of the other; for $\tau=0$ the two variables are statistically independent.

The resulting rank correlation coefficients and the associated $p$ values, are presented in Fig. 10. For each panel, the columns denote the EMD modes (IMFx) and the rows the amplitudes of their envelopes (AMy). The background color of each cell (AM $x$, IMF $y$ ) indicates the rank correlation $\tau$ between IMFy and the AM part of IMF $x$ within the same dataset. The legend of the color encoding is found on the color bar at the bottom of the figure. The associated $p$ values are presented numerically in each cell for the sake of completeness and transparency (Wasserstein and Lazar, 2016). For BOU, CAR, and PAY, IMF6 accounts for the yearly variability in the time series; hence, the correlation matrices are $6 \times 6$ in size. For TAT, the yearly mode is IMF7; thus, in this case the correlation matrix has a size of $7 \times 7$. Two conclusions can be drawn from Fig. 10.

Values of $\tau$ significantly different from zero, shown in red, are recorded in the last column for all stations. These demonstrate a modulation of the amplitude of the components having sub-year timescales, i.e., AM1 to AM5 (and AM6 for TAT), by the yearly IMF, at a statistically significant level $(p \sim 0)$. The effect is most pronounced for PAY, as inferred from the darker red shades (larger rank correlation coefficients).

For the BOU and CAR datasets the first row (AM1) exhibits blue and dark blue cells for IMF3-IMF5 at the statistically significant level. This indicates a negative rank correlation. Similar, but lighter, amplitude modulation is observed on the second row (AM2), but only by IMF4 and IMF5. For the PAY series, this negative rank correlation is greatly reduced for the first row (light blue tones) and is absent in the second row. For TAT no such correlation can be observed. At this point it is interesting to note that, in a similar way to the discussion from Sect. 5.4, the different features of the datasets from Fig. 10 also enable a classification of the local climate experienced by the measuring stations.

It should be mentioned that the amplitude modulation of high-frequency components by lower frequency ones is also found in the sunspots number time series (Chen et al., 2013) and in multiple solar proxies (Kolotkov et al., 2015). The short-term intrinsic periodicities in the solar proxies appear to be indicative of "randomly distributed dynamical processes in the solar atmosphere" that are closely related to the 11-year solar activity and therefore, unsurprisingly, the high-frequency modes are found to be modulated by this latter cycle (Kolotkov et al., 2016). But this phenomenon is not limited to solar activity signals and has also been identified in surface air temperature records (Paluš, 2014), and time series of the sea level (Liu et al., 2007), and may indicate crossscale nonlinear couplings (Paluš, 2014; Huang et al., 2016).

\subsection{The intrinsic timescales of variability in the SSI}

Firstly, the median periods of the IMFs composing the highfrequency band are revisited. It is shown in Fig. 7 that they follow a dyadic repartition that approximates the series dyadic sequence: $3.5,7,14,28$, and 56 days. Such a doubling in frequency in IMFs has been previously reported in astro- 
physical and geophysical signals. When investigating three independent datasets of satellite observations of the (extraterrestrial) total solar irradiance, Lee et al. (2015) consistently find a similar dyadic-scale progression of modes at 13.5, 27, and 54 days, statistically significant within the $95 \%$ level, that correspond to the 27-day solar rotation period and its (sub-)harmonics. Kolotkov et al. (2015) find intrinsic periodicities having an average of $25_{-2}^{+7}$ and $44_{-5}^{+10}$ days in five different solar proxy signals. The mean periods and the associated error bars in (sub-)superscript, estimated at the half-level width of the corresponding probability histogram, were obtained by analyzing the sunspot area for the whole Sun, and for the northern and southern solar hemispheres taken separately, the $10.7 \mathrm{~cm}$ radio flux intensity, and the helioseismic frequency shift. Emery et al. (2011) also find periodicities of $5,7,9,13.5$, and 27 days in different radiation belt, solar wind, geomagnetic, and auroral parameters. Compelling as it may seem, nevertheless, the imprint of a solar rotation signature on ground measurements of the SSI is highly unlikely, as it would imply the existence of hitherto unknown physical mechanisms in Earth's atmosphere (Gerard Thuiller, personal communication, 2015). The amplitudes of the IMFs of the TSI time series and those of the IMFs in the SSI data differ at times by 2 orders of magnitude (e.g., compare Fig. 3 with Fig. 1 in Lee et al., 2015). If the solar rotation signature were to be seen in the IMFs of the SSI this would require the existence of amplifying processes. Stott et al. (2003) and Lockwood and Fröhlich (2007) have studied the possibility of such a mechanism and have concluded that, irrespective of the mechanisms invoked and of the amplification of the solar variability, for the past decades solar forcing is only a minor contributor and thus not able to account for most of the global warming observed in the second half of the 20th century, which could be better explained by an increase in greenhouse gases. Further proof is provided subsequently, this time from a signal theoretical point of view, in support of the view that it is unlikely that the solar rotation signature is captured in measurements of the SSI.

Secondly, in the 100- to 300-day band, two of the stations, BOU and CAR, do not exhibit any variability. For PAY, the support of yearly IMF6 protrudes in this region, although its first quartile rests well below the 200-day mark. As mentioned before, the power of the portion of this IMF that extends into the high-frequency range is very small (not shown). Hence, while not totally devoid of spectral features, this band contains negligible power. A distinct mode is present at TAT in this band, whose median period of 143.2 days somehow seems to continue the dyadic sequence of the previous five modes. Since a similar transitional mode has also been found for two locations in Europe (Bengulescu et al., 2017), presently no explanation in terms of physical processes, such as monsoon rainy seasonality, can be proposed for IMF6 of TAT. These findings are important for the modeling and forecasting of the SSI, as follows. On the one hand, models for BOU and CAR should not contain any power in this band, or should at least filter it out. For TAT, on the other hand, any model attempting to reconstruct the SSI should ensure that the 100- to 300-day region is not a spectral void. In Sect. 5.1, evidence is presented that the spectral band spanning from 2 to 300 days seems to be composed mostly of random realizations of stochastic background processes, which can be modeled following, e.g., Flandrin et al. (2004a), Rilling et al. (2005), Welter and Esquef (2013), and Kolotkov et al. (2016).

Thirdly, the median periods detected around the 1-year mark in all the datasets can be explained by the revolution of the Earth around the Sun and the associated orbital parameters. The interpretation of these components is unambiguous, with one notable exception for the PAY time series, whose IMF6 exhibits mode mixing; i.e., it has a total range that overlaps some of the modes in the high-frequency band. Nevertheless, it will be subsequently shown that it is indeed these components that account for variability at the 1-year timescale.

Lastly, the components indicative of low-frequency variability on timescales greater than 1 year are discussed. The intrinsic timescales found in these IMFs seem to match once more those pertaining to the so-called quasi-biennial oscillations (QBOs) that have been observed in solar activities and proxies with periodicities between 0.6 and 4 years (Bazilevskaya et al., 2015; Kolotkov et al., 2015; Vecchio et al., 2012), as well as in meteorological data like Harrison (2008) who identifies a 1.68-year peak in cloud cover or high-latitude stratospheric temperatures and geopotential heights (Labitzke and Loon, 1988). Nevertheless, within the scope of the current analysis, the interpretation of these lowfrequency variability components as a real, possibly QBOlike, signal is uncertain.

\subsection{The local climate imprint in the IMFs}

It is shown in Sect. 2 that the four measuring stations experience different climates and exhibit differences in terms of $K_{\mathrm{T}}$. Figure 7 shows that the high-frequency band composed of the first five IMFs is very much alike for all stations. This section investigates the possible relationship between local climate and dissimilarities in terms of the repartition of the IMFs with mode number 6 and higher.

It can be noted that the IMF6 for both BOU and CAR has a well-defined period (Fig. 7), with a median of, respectively, 368.2 and 364.3 days and very narrow interquartile range. In addition, for both stations, the IMF6 is the mode having the greatest amplitude, and by far, compared to the other modes (Fig. 8). The IMFs 7 and 8 for CAR have less marked periods, i.e., the interquartile ranges are greater than for IMF6, and the amplitude of each IMF is very small. These observations may be related to the high frequency of cloud-free days seen in Fig. 2 because, in absence of clouds, the variability in the daily mean of SSI is predominantly driven by 
(a) $\mathrm{BOU}$

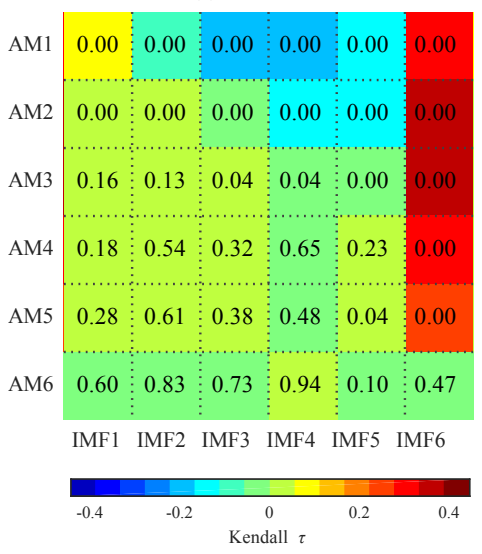

(c) PAY
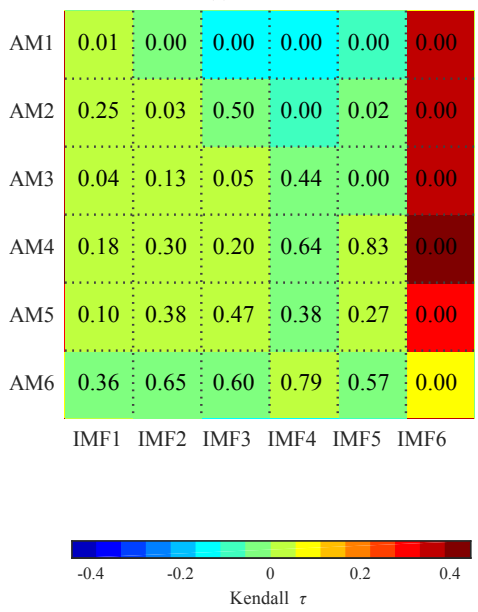

(b) CAR

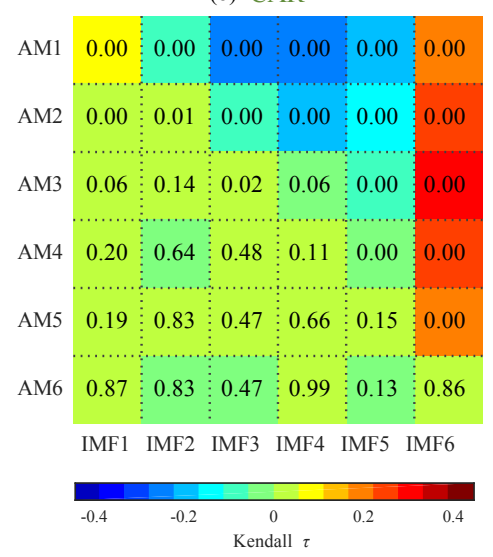

(d) TAT

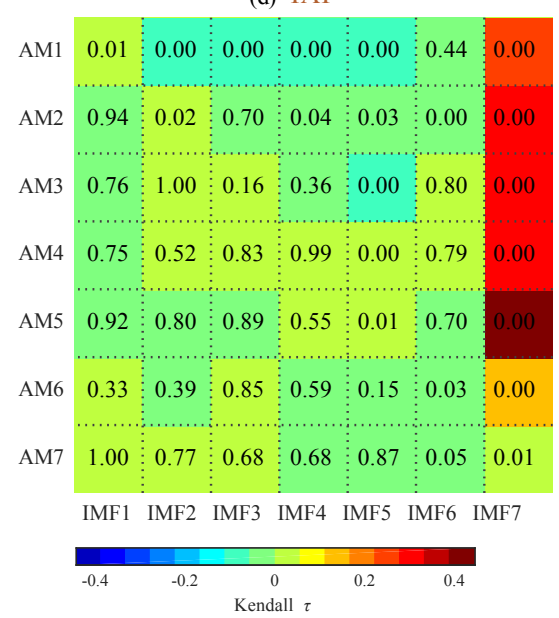

Figure 10. Rank correlations between IMFs and their AM components for BOU (a), CAR (b), PAY (c), and TAT (d). Kendall's rank correlation coefficient $\tau$ is color-coded according to the color bar on the bottom. IMFs run vertically, along the columns, and their AM components run horizontally, along the rows. The numeric values within the cells are the associated $p$ values.

the variability in the solar irradiance received at the top of the atmosphere during the year.

PAY and TAT need four IMFs to account for the lowfrequency variability, i.e., one IMF more than BOU and CAR. IMF6 in PAY has a median period of 356.6 days, close to 1 year (Fig. 7) with a large interquartile range. The median amplitude of the IMF6 is approximately half of that of BOU or CAR (Fig. 8) and the amplitude exhibits large variations. The median amplitude of the IMF7 is similar to that of IMF6 while the period of the IMF7 is well marked with a narrow interquartile range. This may be related to the abundance of the presence of broken clouds that render the SSI signal highly intermittent. This intermittence of the signal could, in turn, explain the mode mixing observed in IMF6 (Huang et al., 2003).

Similar to PAY, TAT also has a low median clearness in$\operatorname{dex} \widetilde{K}_{\mathrm{T}}^{\mathrm{TAT}}=0.51$, which helps explain the presence of a sixth IMF (median period: 143.2 days) between the highfrequency components and the yearly IMF7 (median period:
366.6 days). In other words, the amplitudes of the stochastic components in the sub-year band are higher at TAT than at PAY, or, conversely, there is a lower signal-to-noise ratio of the yearly cycle. Hence, this high power of the background drives the EMD to assign a dedicated intrinsic mode for this region, as opposed to PAY, where the signal in this spectral band is assigned to the yearly IMF through mode mixing.

\section{Conclusion and outlook}

To sum up, the HHT analysis of decennial time series of daily means of measurements of the SSI from distinct BSRN stations has revealed the following: the presence of a highfrequency band (2-100 days) consisting of quasi-stochastic IMFs that have been shown to be amplitude modulated by the yearly cycle; a low-power spectral band in the 100- to 300day region; a well-defined spectral peak at the 1 year mark accounting for the yearly variability; and multiple QBO-like 
components whose character has been, inconclusively, attributed to quasi-stochastic random processes.

This separation of the (quasi-)periodic components of the signal from the apparently random realizations of a stochastic background has been shown to significantly augment accuracy in time-series modeling (Rios and de Mello, 2013). Our findings can be thus directly used to improve models for estimating SSI from satellite images or forecasts of the SSI.

We have shown that the adaptive Hilbert-Huang transform is a versatile tool in analyzing SSI datasets, exhibiting significant nonlinearity and non-stationarity. First, we have employed it to extract the intrinsic modes of variability in the SSI at distinct timescales. Second, the HHT has been used to discriminate between the deterministic yearly cycle and the quasi-stochastic high-frequency components. The same methodology could also be employed on different geophysical signals, such as wind speed time series and river discharge datasets.

When modeling climate processes as dynamical systems with low-frequency oscillations and noise effects, Chekroun et al. (2011) have shown that "even the 'approximately right' noise can help, rather than hinder". Here, we have provided a recipe not only for extracting, but also for characterizing the stochastic high-frequency constituents of long-term time series of the SSI. Indications with respect to modeling these quasi-stochastic components have also been provided. With respect to SSI forecast models, it is exactly this spectral region that is the focus of attention (Ehnberg and Bollen, 2005; Hoff and Perez, 2010; Marquez and Coimbra, 2013). Inman et al. (2013) venture as far as stating that "the accuracy of the solar irradiance forecasting models depends almost exclusively on the ability to forecast the stochastic component". In this light, the recipe for discriminating the realizations of random background processes that we have put forth can be seen as one of the more significant contributions of our paper.

We have also proposed that a classification of the measuring stations according to climate and/or solar insolation conditions may be possible, based on the Hilbert spectral features of the data. Thus, one future research pathway could consist in creating a catalogue of the variability in the solar resource, at different timescales, on a global scale via satellite estimates of the SSI. Current meteorological reanalyses are too noisy in their estimates of the SSI to form the basis for such a catalogue (Boilley and Wald, 2015). In terms of solar power production, the low-frequency variability data would aid with policy and investment decisions, while shortterm variability would be of interest from a monitoring, operations, and engineering perspective.

Code availability. The software used for this study, comprising general EMD and Hilbert spectral analysis routines, is publicly available online.
- The fast EMD routine used in this study is provided by Wang et al. (2014) and can be downloaded at http://rcada.ncu.edu.tw/FEEMD.rar.

- Methods pertaining to Hilbert spectral analysis are part of a general HHT toolkit provided by Wu and Huang (2009) and can be downloaded at http://rcada.ncu.edu.tw/Matlab\%20runcode.zip.

- The code for the ICEEMD(AN) algorithm (Colominas et al., 2014) is provided by María Eugenia Torres on her personal web page and can be downloaded at http://bioingenieria.edu.ar/grupos/ldnlys/metorres/metorres_ files/ceemdan_v2014.m.

Data availability. The raw BSRN datasets employed in this study are made available by König-Langlo et al. (2015). Zip archives containing the data can be found at https://doi.pangaea.de/10.1594/ PANGAEA.852720.

Author contributions. All authors contributed equally to this work.

Competing interests. The authors declare that they have no conflict of interest.

Acknowledgements. The authors wish to thank Patrick Flandrin from École Normale Supérieure de Lyon, France, and Gerard Thuiller from Laboratoire Atmosphères, Milieux, Observations Spatiales in Guyancourt, France, for the fruitful conversations and their insightful comments that sparked the development of this study. Dmitrii Kolotkov from the University of Warwick, United Kingdom, is also acknowledged for the personal communications pertaining to the stochastic nature of the high-frequency variability band. The authors thank all ground station operators of the BSRN network for their valuable measurements and the Alfred Wegener Institute (AWI) for hosting the BSRN website.

Edited by: Ioulia Tchiguirinskaia

Reviewed by: two anonymous referees

\section{References}

Alberti, T., Lepreti, F., Vecchio, A., Bevacqua, E., Capparelli, V., and Carbone, V.: Natural periodicities and Northern Hemisphere-Southern Hemisphere connection of fast temperature changes during the last glacial period: EPICA and NGRIP revisited, Clim. Past, 10, 1751-1762, https://doi.org/10.5194/cp10-1751-2014, 2014.

Alberti, T., Piersanti, M., Vecchio, A., De Michelis, P., Lepreti, F., Carbone, V., and Primavera, L.: Identification of the different magnetic field contributions during a geomagnetic storm in magnetospheric and ground observations, Ann. Geophys., 34, 10691084, https://doi.org/10.5194/angeo-34-1069-2016, 2016.

Bazilevskaya, G., Broomhall, A.-M., Elsworth, Y., and Nakariakov, V.: A combined analysis of the observational aspects 
of the quasi-biennial oscillation in solar magnetic activity, in: The Solar Activity Cycle, Springer, 186, 359-386, https://doi.org/10.1007/978-1-4939-2584-1_12, 2015.

Beer, J., Vonmoos, M., and Muscheler, R.: Solar variability over the past several millennia, Space Sci. Rev., 125, 67-79, https://doi.org/10.1007/s11214-006-9047-4, 2006.

Bengulescu, M., Blanc, P., Boilley, A., and Wald, L.: Do modelled or satellite-based estimates of surface solar irradiance accurately describe its temporal variability?, Adv. Sci. Res., 14, 35-48, https://doi.org/10.5194/asr-14-35-2017, 2017.

Bengulescu, M., Blanc, P., and Wald, L.: On the temporal variability of the surface solar radiation by means of spectral representations, Adv. Sci. Res., 13, 121-127, https://doi.org/10.5194/asr13-121-2016, 2016a.

Bengulescu, M., Blanc, P., and Wald, L.: Characterizing temporal variability in measurements of surface solar radiation and its dependence on climate, Energy Procedia, 97, 164-171, https://doi.org/10.1016/j.egypro.2016.10.045, 2016b.

Blanc, P., Coulaud, C., and Wald, L.: Yearly changes in surface solar radiation in New Caledonia, Adv. Sci. Res., 12, 1-4, https://doi.org/10.5194/asr-12-1-2015, 2015.

Boilley, A. and Wald, L.: Comparison between meteorological reanalyses from ERA-Interim and MERRA and measurements of daily solar irradiation at surface, Renew. Energ., 75, 135-143, https://doi.org/10.1016/j.renene.2014.09.042, 2015.

Bojinski, S., Verstraete, M., Peterson, T. C., Richter, C., Simmons, A., and Zemp, M.: The concept of Essential Climate Variables in support of climate research, applications, and policy, B. Am. Meteorol. Soc., 95, 1431-1443, https://doi.org/10.1175/BAMSD-13-00047.1, 2014.

BSRN station listing, available at: https://www.pangaea.de/ ddi?request=bsrn/BSRNEvent $\&$ format $=$ html\&title $=B S R N+$ Stations, last access: 9 December 2015.

Calif, R., Schmitt, F. G., Huang, Y., and Soubdhan, T.: Intermittency study of high frequency global solar radiation sequences under a tropical climate, Sol. Energy, 98, 349-365, https://doi.org/10.1016/j.solener.2013.09.018, 2013.

Chekroun, M. D., Kondrashov, D., and Ghil, M.: Predicting stochastic systems by noise sampling, and application to the El NiñoSouthern Oscillation, P. Natl. Acad. Sci. USA, 108, 1176611771, https://doi.org/10.1073/pnas.1015753108, 2011.

Chen, X., Wang, M., Zhang, Y., Feng, Y., Wu, Z., and Huang, N. E.: Detecting signals from data with noise: theory and applications, J. Atmos. Sci., 70, 1489-1504, https://doi.org/10.1175/JAS-D12-0213.1, 2013.

Cohen, L.: Time-frequency distributions-a review, Proceedings of the IEEE, 77, 941-981, https://doi.org/10.1109/5.30749, 1989.

Colominas, M. A., Schlotthauer, G., Torres, M. E., and Flandrin, P.: Noise-assisted EMD methods in action, Advances in Adaptive Data Analysis, 4, 1250025, https://doi.org/10.1142/S1793536912500252, 2012.

Colominas, M. A., Schlotthauer, G., and Torres, M. E.: Improved complete ensemble EMD: A suitable tool for biomedical signal processing, Biomed. Signal Proces., 14, 19-29, https://doi.org/10.1016/j.bspc.2014.06.009, 2014.

Coskun, C., Oktay, Z., and Dincer, I.: Estimation of monthly solar radiation distribution for solar energy system analysis, Energy, 36, 1319-1323, https://doi.org/10.1016/j.energy.2010.11.009, 2011.
Duffy, D. G.: The application of Hilbert-Huang transforms to meteorological datasets, J. Atmos. Ocean. Tech., 21, 599-611, https://doi.org/10.1175/15200426(2004)021<0599:TAOHTT>2.0.CO;2, 2004.

Ehnberg, J. S. and Bollen, M. H.: Simulation of global solar radiation based on cloud observations, Sol. Energy, 78, 157-162, https://doi.org/10.1016/j.solener.2004.08.016, 2005.

Emery, B. A., Richardson, I. G., Evans, D. S., Rich, F. J., and Wilson, G. R.: Solar rotational periodicities and the semiannual variation in the solar wind, radiation belt, and aurora, Sol. Phys., 274, 399-425, https://doi.org/10.1007/s11207-011-9758-x, 2011.

Flandrin, P. and Gonçalvès, P.: Empirical mode decompositions as data-driven wavelet-like expansions, Int. J. Wavelets Multi., 2, 477-496, https://doi.org/10.1142/S0219691304000561, 2004.

Flandrin, P., Gonçalvès, P., and Rilling, G.: EMD equivalent filter banks, from interpretation to applications, in: Hilbert-Huang Transform and Its Applications, World Scientific Pub Co Pte Lt, 57-74, https://doi.org/10.1142/9789812703347_0003, 2005.

Flandrin, P., Rilling, G., and Gonçalvès, P.: Empirical mode decomposition as a filter bank, IEEE Signal Proc. Let., 11, 112-114, https://doi.org/10.1109/LSP.2003.821662, 2004a.

Flandrin, P., Gonçalvès, P., and Rilling, G.: Detrending and denoising with empirical mode decompositions, in: 2004 12th European Signal Processing Conference, 6-10 September 2004, Vienna, Austria, 1581-1584, 2004b.

Franzke, C.: Multi-scale analysis of teleconnection indices: climate noise and nonlinear trend analysis, Nonlin. Processes Geophys., 16, 65-76, https://doi.org/10.5194/npg-16-65-2009, 2009.

Franzke, C.: Nonlinear trends, long-range dependence, and climate noise properties of surface temperature, J. Climate, 25, 41724183, https://doi.org/10.1175/JCLI-D-11-00293.1, 2012.

Gabor, D.: Theory of communication. Part 1: The analysis of information, Journal of the Institution of Electrical Engineers Part III: Radio and Communication Engineering, 93, 429-441, https://doi.org/10.1049/ji-3-2.1946.0074, 1946.

Harrison, R. G.: Discrimination between cosmic ray and solar irradiance effects on clouds, and evidence for geophysical modulation of cloud thickness, P. Roy. Soc. Lond. A Mat., 464, 25752590, https://doi.org/10.1098/rspa.2008.0081, 2008.

Hathaway, D. H.: The solar cycle, Living Rev. Sol. Phys., 12, 1-87, https://doi.org/10.1007/lrsp-2015-4, 2015.

Hoff, T. E. and Perez, R.: Quantifying PV power output variability, Sol. Energy, 84, 1782-1793, https://doi.org/10.1016/j.solener.2010.07.003, 2010.

Huang, N. E. and Shen, S. S. P.: Hilbert-Huang Transform and Its Applications, 2nd Edn., World Scientific, https://doi.org/10.1142/9789814508247_fmatter, 2014.

Huang, N. E. and Wu, Z.: A review on Hilbert-Huang transform: Method and its applications to geophysical studies, Rev. Geophys., 46, RG2006, https://doi.org/10.1029/2007RG000228, 2008.

Huang, N. E., Shen, Z., Long, S. R., Wu, M. C., Shih, H. H., Zheng, Q., Yen, N.-C., Tung, C. C., and Liu, H. H.: The empirical mode decomposition and the Hilbert spectrum for nonlinear and nonstationary time series analysis, P. Roy. Soc. Lond. A Mat., 454, 903-995, https://doi.org/10.1098/rspa.1998.0193, 1998.

Huang, N. E., Wu, M.-L. C., Long, S. R., Shen, S. S. P., Qu, W., Gloersen, P., and Fan, K. L.: A confidence limit for the empirical mode decomposition and Hilbert spec- 
tral analysis, P. Roy. Soc. Lond. A Mat., 459, 2317-2345, https://doi.org/10.1098/rspa.2003.1123, 2003.

Huang, N. E., Wu, Z., Long, S. R., Arnold, K. C., Chen, X., and Blank, K.: On instantaneous frequency, Advances in Adaptive Data Analysis, 1, 177-229, https://doi.org/10.1142/S1793536909000096, 2009.

Huang, N. E., Chen, X., Lo, M.-T., and Wu, Z.: On Hilbert spectral representation: a true time-frequency representation for nonlinear and nonstationary data, Advances in Adaptive Data Analysis, 3, 63-93, https://doi.org/10.1142/S1793536911000659, 2011.

Huang, N. E., Hu, K., Yang, A. C., Chang, H.-C., Jia, D., Liang, W.K., Yeh, J. R., Kao, C.-L., Juan, C.-H., Peng, C. K., Meijer, J. H., Wang, Y.-H., Long, S. R., and Wu, Z.: On Holo-Hilbert spectral analysis: a full informational spectral representation for nonlinear and non-stationary data, Philos. T. R. Soc. A, 374, 20150206 , https://doi.org/10.1098/rsta.2015.0206, 2016.

Inman, R. H., Pedro, H. T., and Coimbra, C. F.: Solar forecasting methods for renewable energy integration, Prog. Energ. Combust., 39, 535-576, https://doi.org/10.1016/j.pecs.2013.06.002, 2013.

Kendall, M. G.: A new measure of rank correlation, BIOMETRIKA, 30, 81-93, https://doi.org/10.1093/biomet/30.12.81, 1938.

Kolotkov, D., Broomhall, A.-M., and Nakariakov, V.: HilbertHuang transform analysis of periodicities in the last two solar activity cycles, Mon. Not. R. Astron. Soc., 451, 4360-4367, https://doi.org/10.1093/mnras/stv1253, 2015.

Kolotkov, D., Anfinogentov, S. A., and Nakariakov, V. M.: Empirical mode decomposition analysis of random processes in the solar atmosphere, Astron. Astrophys., 592, A153, https://doi.org/10.1051/0004-6361/201628306, 2016.

König-Langlo, G., Driemel, A., Raffel, B., and Sieger, R.: BSRN snapshot 2015-09, links to zip archives, PANGAEA, https://doi.org/10.1594/PANGAEA.852720, 2015.

Kottek, M., Grieser, J., Beck, C., Rudolf, B., and Rubel, F.: World map of the Köppen-Geiger climate classification updated, Meteorol. Z., 15, 259-263, https://doi.org/10.1127/09412948/2006/0130, 2006.

Labitzke, K. and Loon, H. V.: Associations between the 11-year solar cycle, the QBO and the atmosphere. Part I: the troposphere and stratosphere in the northern hemisphere in winter, J. Atmos. Terr. Phys., 50, 197-206, https://doi.org/10.1016/00219169(88)90068-2, 1988.

Lauret, P., Perez, R., Aguiar, L. M., Tapachès, E., Diagne, H. M., and David, M.: Characterization of the intraday variability regime of solar irradiation of climatically distinct locations, Sol. Energy, 125, 99-110, https://doi.org/10.1016/j.solener.2015.11.032, 2016.

Lee, J. N., Cahalan, R. F., and Wu, D. L.: The 27-day rotational variations in total solar irradiance observations: From SORCE/TIM, ACRIMSAT/ACRIM III, and SOHO/VIRGO, J. Atmos. Sol.-Terr. Phy., 132, 64-73, https://doi.org/10.1016/j.jastp.2015.07.001, 2015.

Lee, T. and Ouarda, T. B. M. J.: Prediction of climate nonstationary oscillation processes with empirical mode decomposition, J. Geophys. Res., 116, D06107, https://doi.org/10.1029/2010JD015142, 2011.
Liu, Y., San Liang, X., and Weisberg, R. H.: Rectification of the bias in the wavelet power spectrum, J. Atmos. Ocean. Tech., 24, 2093-2102, https://doi.org/10.1175/2007JTECHO511.1, 2007.

Lockwood, M. and Fröhlich, C.: Recent oppositely directed trends in solar climate forcings and the global mean surface air temperature, P. Roy. Soc. Lond. A Mat., 463, 2447-2460, https://doi.org/10.1098/rspa.2007.1880, 2007.

Marquez, R. and Coimbra, C. F.: Proposed metric for evaluation of solar forecasting models, J. Sol. Energ.-T. ASME, 135, 011016, https://doi.org/10.1115/1.4007496, 2013.

Medvigy, D. and Beaulieu, C.: Trends in daily solar radiation and precipitation coefficients of variation since 1984, J. Climate, 25, 1330-1339, https://doi.org/10.1175/2011JCLI4115.1, 2012.

Moghtaderi, A., Flandrin, P., and Borgnat, P.: Trend filtering via empirical mode decompositions, Comput. Stat. Data An., 58, 114 126, https://doi.org/10.1016/j.csda.2011.05.015, 2013.

Nagovitsyn, Y. A.: A nonlinear mathematical model for the solar cyclicity and prospects for reconstructing the solar activity in the past, Astron. Lett.+, 23, 742-748, http://tinyurl.com/gm6c4u9, 1997.

Ohmura, A., Gilgen, H., Hegner, H., Müller, G., Wild, M., Dutton, E. G., Forgan, B., Fröhlich, C., Philipona, R., Heimo, A., König-Langlo, G., McArthur, B., Pinker, R., Whitlock, C. H., and Dehne, K.: Baseline surface radiation network (BSRN/WCRP): new precision radiometry for climate research, B. Am. Meteorol. Soc., 79, 2115-2136, https://doi.org/10.1175/15200477(1998)079<2115:BSRNBW>2.0.CO;2, 1998.

Pachauri, R. K., Allen, M., Barros, V., et al.: Climate Change 2014: Synthesis Report. Contribution of Working Groups I, II and III to the Fifth Assessment Report of the Intergovernmental Panel on Climate Change, edited by: Pachauri, R. and Meyer, L., Geneva, Switzerland, IPCC, 151 pp., available at: http://epic.awi. de/37530/ (last access: 22 January 2018), 2014.

Paluš, M.: Multiscale atmospheric dynamics: crossfrequency phase-amplitude coupling in the air temperature, Phys. Rev. Lett., 112, 078702, https://doi.org/10.1103/PhysRevLett.112.078702, 2014.

Rilling, G., Flandrin, P., and Gonçalvès, P.: On empirical mode decomposition and its algorithms, in: Proceedings of IEEEEURASIP Workshop on Nonlinear Signal and Image Processing NSIP-03, Grado (Italy), 3, 8-11, available at: http://tinyurl.com/ k3z2yv3 (last access: 22 January 2018), 2003.

Rilling, G., Flandrin, P., and Gonçalves, P.: Empirical mode decomposition, fractional Gaussian noise and Hurst exponent estimation, in: Acoustics, Speech, and Signal Processing, 2005. Proceedings. (ICASSP '05). IEEE International Conference on, 2323 March 2005, Philadelphia, PA, USA, IEEE, 4, iv/489-iv/492, https://doi.org/10.1109/ICASSP.2005.1416052, 2005.

Rios, R. A. and de Mello, R. F.: Improving time series modeling by decomposing and analyzing stochastic and deterministic influences, Signal Process., 93, 3001-3013, https://doi.org/10.1016/j.sigpro.2013.04.017, 2013.

Rios, R. A. and de Mello, R. F.: Applying Empirical Mode Decomposition and mutual information to separate stochastic and deterministic influences Signal Processingembedded in signals, Signal Process., 118, 159-176, https://doi.org/10.1016/j.sigpro.2015.07.003, 2016.

Rios, R. A., Parrott, L., Lange, H., and de Mello, R. F.: Estimating determinism rates to detect patterns in 
geospatial datasets, Remote Sens. Environ., 156, 11-20, https://doi.org/10.1016/j.rse.2014.09.019, 2015.

Roesch, A., Wild, M., Ohmura, A., Dutton, E. G., Long, C. N., and Zhang, T.: Assessment of BSRN radiation records for the computation of monthly means, Atmos. Meas. Tech., 4, 339-354, https://doi.org/10.5194/amt-4-339-2011, 2011.

Schlotthauer, G., Torres, M. E., Rufiner, H. L., and Flandrin, P.: EMD of Gaussian white noise: effects of signal length and sifting number on the statistical properties of intrinsic mode functions, Advances in Adaptive Data Analysis, 1, 517-527, https://doi.org/10.1142/S1793536909000217, 2009.

Schroedter-Homscheidt, M., Delamare, C., Heilscher, G., Heinemann, D., Hoyer, C., Meyer, R., Toggweiler, P., Wald, L., and Zelenka, A.: The ESA-ENVISOLAR project: experience on the commercial use of Earth observation based solar surface irradiance measurements for energy business purposes, in: Solar Energy Resources Management for Electricity Generation, edited by: Dunlop, E. D., Wald, L., and Šúri, M., Nova Science Publishers, 111-124, http://tinyurl.com/hpf8d5g, 2006.

Solé, J., Turiel, A., and Llebot, J. E.: Using empirical mode decomposition to correlate paleoclimatic time-series, Nat. Hazards Earth Syst. Sci., 7, 299-307, https://doi.org/10.5194/nhess7-299-2007, 2007.

Stott, P. A., Jones, G. S., and Mitchell, J. F.: Do models underestimate the solar contribution to recent climate change?, J. Climate, 16, 4079-4093, https://doi.org/10.1175/15200442(2003)016<4079:DMUTSC>2.0.CO;2, 2003.

Tary, J. B., Herrera, R. H., Han, J., and van der Baan, M.: Spectral estimation - What is new? What is next?, Rev. Geophys., 52, 723-749, https://doi.org/10.1002/2014RG000461, 2014.

Torrence, C. and Compo, G. P.: A practical guide to wavelet analysis, B. Am. Meteorol. Soc., 79, 61-78, https://doi.org/10.1175/15200477(1998)079<0061:APGTWA>2.0.CO;2, 1998.

Torres, M. E., Colominas, M. A., Schlotthauer, G., and Flandrin, P.: A complete ensemble empirical mode decomposition with adaptive noise, in: Acoustics, Speech and Signal Processing (ICASSP), 2011 IEEE International Conference on, 22-27 May 2011, Prague, Czech Republic, IEEE, 4144-4147, 2011.

Trenberth, K. E., Fasullo, J. T., and Kiehl, J.: Earth's global energy budget, B. Am. Meteorol. Soc., 90, 311-323, https://doi.org/10.1175/2008BAMS2634.1, 2009.

Vecchio, A., Capparelli, V., and Carbone, V.: The complex dynamics of the seasonal component of USA's surface temperature, Atmos. Chem. Phys., 10, 9657-9665, https://doi.org/10.5194/acp10-9657-2010, 2010.
Vecchio, A., Laurenza, M., Meduri, D., Carbone, V., and Storini, M.: The dynamics of the solar magnetic field: polarity reversals, butterfly diagram, and quasi-biennial oscillations, Astrophys. J., 749, 27, https://doi.org/10.1088/0004-637X/749/1/27, 2012.

Wahab, M. A., El-Metwally, M., Hassan, R., Lefevre, M., Oumbe, A., and Wald, L.: Assessing surface solar irradiance and its long-term variations in the northern Africa desert climate using Meteosat images, Int. J. Remote Sens., 31, 261-280, https://doi.org/10.1080/01431160902882645, 2010.

Wang, G., Chen, X.-Y., Qiao, F.-L., Wu, Z., and Huang, N. E.: On intrinsic mode function, Advances in Adaptive Data Analysis, 2, 277-293, https://doi.org/10.1142/S1793536910000549, 2010.

Wang, Y.-H., Yeh, C.-H., Young, H.-W. V., Hu, K., and Lo, M.-T.: On the computational complexity of the empirical mode decomposition algorithm, Physica A, 400, 159-167, https://doi.org/10.1016/j.physa.2014.01.020, 2014.

Wasserstein, R. L. and Lazar, N. A.: The ASA's statement on $p$ values: context, process, and purpose, Am. Stat., 70, 129-133, https://doi.org/10.1080/00031305.2016.1154108, 2016.

Welter, G. S. and Esquef, P. A. A.: Multifractal analysis based on amplitude extrema of intrinsic mode functions, Phys. Rev. E, 87, 032916, https://doi.org/10.1103/PhysRevE.87.032916, 2013.

Wu, Z. and Huang, N. E.: A study of the characteristics of white noise using the empirical mode decomposition method, P. Roy. Soc. Lond. A Mat., 460, 1597-1611, https://doi.org/10.1098/rspa.2003.1221, 2004.

$\mathrm{Wu}, \mathrm{Z}$. and Huang, N. E.: Ensemble empirical mode decomposition: a noise-assisted data analysis method, Advances in Adaptive Data Analysis, 01, 1-41, https://doi.org/10.1142/S1793536909000047, 2009.

Wu, Z. and Huang, N. E.: On the filtering properties of the empirical mode decomposition, Advances in Adaptive Data Analysis, 2, 397-414, https://doi.org/10.1142/S1793536910000604, 2010.

Wu, Z., Huang, N. E., and Chen, X.: Some considerations on physical analysis of data, Advances in Adaptive Data Analysis, 3, 95113, https://doi.org/10.1142/S1793536911000660, 2011.

Yordanov, G. H., Saetre, T. O., and Midtgard, O.-M.: 100-millisecond resolution for accurate overirradiance measurements, IEEE J. Photovolt., 3, 1354-1360, https://doi.org/10.1109/JPHOTOV.2013.2264621, 2013.

Zeng, Z., Yang, H., Zhao, R., and Meng, J.: Nonlinear characteristics of observed solar radiation data, Sol. Energy, 87, 204-218, https://doi.org/10.1016/j.solener.2012.10.019, 2013. 